\title{
REDUCTION TYPE OF SMOOTH PLANE QUARTICS
}

\author{
REYNALD LERCIER, QING LIU, ELISA LORENZO GARCÍA, AND CHRISTOPHE RITZENTHALER
}

\begin{abstract}
Let $C / K$ be a smooth plane quartic over a discrete valuation field. We characterize the type of reduction (i.e. smooth plane quartic, hyperelliptic genus 3 curve or bad) over $K$ in terms of the existence of a special plane quartic model and, over $\bar{K}$, in terms of the valuations of certain algebraic invariants of $C$ when the characteristic of the residue field is not 2, 3,5 or 7 . On the way, we gather several results of general interest on geometric invariant theory over an arbitrary ring $R$ in the spirit of [Ses77]. For instance when $R$ is a discrete valuation ring, we show the existence of a homogeneous system of parameters over $R$. We exhibit explicit ones for ternary quartic forms under the action of $\mathrm{SL}_{3, R}$ depending only on the characteristic $p$ of the residue field. We illustrate our results with the case of Picard curves for which we give simple criteria for the type of reduction.
\end{abstract}

\section{INTRODUCTION AND MAIN RESULTS}

Let $K$ be a discrete valuation field with valuation $v$, valuation ring $\mathcal{O}$ and a uniformizer $\pi$. Let $k=\mathcal{O} /\langle\pi\rangle$ be the residue field of characteristic $p \geq 0$. When $F$ is an integral polynomial, i.e. with coefficients in $\mathcal{O}$, we denote by $\bar{F}$ its reduction modulo $\pi$. A smooth projective geometrically connected curve of genus 3 is either a plane quartic or a hyperelliptic curve depending on whether the canonical divisor is very ample or not. Let $C / K$ be a smooth plane quartic.

- We say that $C$ has good (resp. good quartic, resp. good hyperelliptic) reduction over $K$ if $C$ has a projective smooth model $\mathcal{C}$ over $\mathcal{O}$ (resp. whose special fiber is a smooth plane quartic, resp. a hyperelliptic curve).

- We say that $C$ has potentially good (resp. potentially good quartic, resp. potentially good hyperelliptic) reduction if for some finite separable extension $\left(K^{\prime}, v^{\prime}\right)$ of $(K, v), C_{K^{\prime}} / K^{\prime}$ has good (resp. good quartic, resp. good hyperelliptic) reduction over $K^{\prime}$.

- We say that $C$ has bad reduction over $K$ if it does not have good reduction over $K$.

Note that the type of the potential reduction does not depend on the choice of $\left(K^{\prime}, v^{\prime}\right)$ and is given by the type of reduction of the stable model of $C$ over some finite separable extension of $K$. Hence, in the following, the expression "after a possible finite extension of $K$ ", or potentially, means that we are allowed to take a finite extension of $K$ and still call $K, \mathcal{O}, v, \pi$ the corresponding notions. Let us point out already here that when using the adjective (semi)-stable alone, we always mean in the Deligne-Mumford sense of (semi)-stability. Otherwise, while talking later about Geometric Invariant Theory (GIT) stability, we will always write GIT-(semi-)stable.

Given such a curve $C / K$, we want to determine the reduction type of $C$ among the above possibilities. In the first two cases, we also look for an explicit equation of the special fiber. In the first case, it

Date: August 4, 2020.

2010 Mathematics Subject Classification. 11G20, 14Q05, 14D10, 14D20, 14H25.

Key words and phrases. smooth plane quartic, reduction, hyperelliptic, invariants, valuation.

The authors would like to thank Jeroen Sijsling for his careful reading of an earlier version of the paper, Matthieu Romagny for useful references, Bianca Viray and Armand Brumer for inspiring discussions, and the referee for his/her comments improving the presentation of the manuscript. The second named author is partly supported by Xiamen University and the NSFC grant no. 11031004. 
will be again a smooth plane quartic over $k$, whereas in the second case it will be isomorphic over $\bar{k}$ to $y^{2}=f(x, z)$ where $f$ is a binary octic with no multiple roots.

Example 1.1. Let us consider the plane smooth quartic $C / \mathbb{Q}: F=0$ where

$$
F=\left(x_{2}+x_{3}\right) x_{1}^{3}-\left(2 x_{2}^{2}+x_{3} x_{2}\right) x_{1}^{2}+\left(x_{2}^{3}-x_{3} x_{2}^{2}+2 x_{3}^{2} x_{2}-x_{3}^{3}\right) x_{1}-2 x_{3}^{2} x_{2}^{2}+3 x_{3}^{3} x_{2} .
$$

This curve is an equation of $X_{n s}(13) \simeq X_{s}(13) \simeq X_{0}^{+}(169)$ which is studied in [BDM ${ }^{+} 19$, Cor.6.8]. By using a general result from [Edi90], they prove that this curve has good quartic reduction away from 13 and potentially good hyperelliptic reduction at 13 after a ramified extension of degree 84 . By using the characterizations we are giving in this article and our implementations, we can automatically check that the curve has potentially good hyperelliptic reduction at 13 and that the special fiber is the hyperelliptic curve of affine equation $y^{2}=x^{7}-1$ (see Example 5.13).

The most complicated task will be to distinguish good hyperelliptic reduction from bad reduction. By identifying a plane quartic with its 15 coefficients up to a multiplicative constant, we can view the set $\boldsymbol{X}$ of smooth plane quartics as a subset of $\mathbb{P}^{14}$. Since isomorphisms between smooth plane quartics are linear, $\boldsymbol{X} / \mathrm{SL}_{3}(\bar{K})$ gives a description of the locus $\boldsymbol{M}_{3}^{\text {qrt }}$ of plane quartics inside the moduli space $\boldsymbol{M}_{3}$ of all curves of genus 3. Considering the locus of hyperelliptic curves as "a boundary" of the locus of $\boldsymbol{M}_{3}^{\text {qrt }}$ inside $\boldsymbol{M}_{3}$, the challenge is to understand whether a family of plane smooth quartics has limit in this boundary. Similar situations have been studied to describe the boundary of the compactification of $\boldsymbol{M}_{3}$ and there is a rich literature on this subject [Art09, Gla79, Has99, HL10, Kan00, Kon00, Mum77, Yuk86]. Surprisingly, the mentioned problem has never been fully addressed before (still, see [HLP00, Prop.14 and Prop.15] in the case of Ciani quartics). Known results about the relations between plane quartics and hyperelliptic genus 3 curves are basically contained in the following proposition [Cle80, p.156], [Har87], [HM98, p.134].

Proposition 1.2. Suppose $p \neq 2$. Let $s>0$ be an integer, $G \in \mathcal{O}\left[x_{1}, x_{2}, x_{3}\right]$ be a primitive (i.e. the gcd of its coefficients is 1) quartic form and $Q \in \mathcal{O}\left[x_{1}, x_{2}, x_{3}\right]$ be a primitive quadratic form. Let us assume that $\bar{Q}$ is irreducible and that $\bar{Q}=0$ intersects $\bar{G}=0$ transversely in 8 distinct $\bar{k}$-points. Let us denote $\mathcal{C} / \mathcal{O}$ the subscheme of the weighted projective space $\mathbb{P}^{(1,1,1,2)}$ defined by

$$
\left\{\begin{array}{l}
y^{2}+G=0, \\
\pi^{s} y-Q=0 .
\end{array}\right.
$$

Then the generic fiber is isomorphic to the plane smooth quartic $C / K: Q^{2}+\pi^{2 s} G=0$ which has good hyperelliptic reduction. The special fiber of $\mathcal{C}$ is isomorphic to the double cover of $\bar{Q}=0$ ramified over the 8 distinct intersection $\bar{k}$-points of $\bar{Q}=0$ with $\bar{G}=0$.

This motivates the following definitions.

Definition 1.3. Let $C / K$ be a plane smooth quartic. When $p \neq 2$, we say that $C$ admits a toggle model if there exist an even integer $s>0$, a primitive quartic form $G \in \mathcal{O}\left[x_{1}, x_{2}, x_{3}\right]$ and a primitive quadric $Q \in \mathcal{O}\left[x_{1}, x_{2}, x_{3}\right]$ with $\bar{Q}$ irreducible such that $Q^{2}+\pi^{2 s} G=0$ is $K$-isomorphic to $C$. If, moreover, $\bar{Q}=0$ intersects $\bar{G}=0$ transversely in 8 distinct $\bar{k}$-points, the model $Q^{2}+\pi^{2 s} G=0$ is a good toggle model of $C$. We refer to Theorem 2.9 for the definition when $p=2$.

When $C / K$ admits a plane quartic model over $\mathcal{O}$ which special fiber is smooth then obviously this plane model is a stable model of $C$. When $C$ admits a good toggle model, it has good hyperelliptic 
reduction and (1.1) is a stable (actually smooth) model of $C$. The converse holds as well, this is the main result of Section 2 .

Theorem 1.4 (See Theorems 2.8 and 2.9). Let $K$ be a discrete valuation field with residue field of characteristic $p \geq 0$. Let $C / K$ be a smooth plane quartic. Then $C$ admits good hyperelliptic reduction over $K$ if and only if $C$ has a good toggle model over $K$.

By using this result, the methods of [Kol97] and [ES20] and some ingredients from invariant theory, the question of the reduction type (good quartic, good hyperelliptic or bad) over $K$ of a smooth quartic may be answered (see remark 5.2).

We turn now to the determination of the type when one allows extensions of $K$. In the spirit of the work of [Liu93] on genus 2, we aim at getting a characterization of the potential reduction type in terms of the valuations of certain invariants of $C / K$ under the action of $\mathrm{SL}_{3}(\bar{K})$. Let us start by recalling some results on the homogeneous algebra of invariants of ternary quartic forms.

In [Dix87], Dixmier worked out a list of 7 homogeneous polynomial invariants for the equivalence of ternary quartic forms under the action of $\mathrm{SL}_{3}(\mathbb{C})$, denoted $I_{3}, I_{6}, I_{9}, I_{12}, I_{15}, I_{18}$ and $I_{27}$. They form a Homogeneous System Of Parameters (HSOP) for the algebra of invariants $\mathcal{A}$ over $\mathbb{C}$, which means that the radical of the ideal they generate is equal to the irrelevant ideal of the homogeneous algebra $\mathcal{A}$ (see Definition 3.7 and remark 3.9). Later, this list is completed by Ohno ([Ohn07, Theorem 4.1], see also [Els15]) into a list of 13 homogeneous generators of $\mathcal{A}$ as $\mathbb{C}$-algebra. Theses invariants are defined over $\mathbb{Z}\left[\frac{1}{2 \cdot 3}\right]$, and are now called the Dixmier-Ohno invariants. We denote DO the list of these invariants, $\underline{\mathrm{DO}}(F) \in K^{13}$ their values at a ternary quartic form $F$ and when at least one of these values is not zero, we denote $\mathrm{DO}(F)$ the corresponding point in the weighted projective space with weights $3,6,9,9,12,12,15,15,18,18,21,21,27$. They determine the isomorphism classes of smooth quartic curves over $\mathbb{C}$ in the sense that two smooth quartics $C_{i}: F_{i}=0$ are isomorphic over $\mathbb{C}$ if and only if $\operatorname{DO}\left(F_{1}\right)=$ $\operatorname{DO}\left(F_{2}\right)$.

This result over $\mathbb{C}$ is clearly not sufficient for our purposes as we want to deal with fields of arbitrary characteristic and with reduction properties. Fortunately, Seshadri in [Ses77] has generalized the Geometric Invariant Theory (GIT) of Mumford over an arbitrary ring $R$. For the convenience of the reader, we include in Section 3.1 some general results on this topic and proofs we were unable to find in the literature. We also prove, under some assumptions on $R^{1}$, that there exists a HSOP over $R$, i.e. a finite list of invariants defined over $R$ being a HSOP after base change to all residue fields of $R$. It is a bit of a surprise as one knows that there exist invariants over fields of small characteristics which do not come from reducing invariants in characteristic 0 (see [Bas15] for binary forms and remark 4.6 for ternary quartics). In principle, one should be able to exhibit a HSOP over $\mathbb{Z}$, but in practice the degrees may be too large to be practical. This is why in Theorem 4.1, we determine explicit HSOP over $\mathcal{O}$ for ternary quartic forms which depend only on the characteristic of the residue field. This is achieved in all characteristics but 3, for which we only have a conjectural HSOP. (see Theorem 4.3). Note also that even for some large characteristics, like 523, the Dixmier invariants alone may not be a HSOP unlike in characteristic 0 . With the normalization of [GKZ94, p.426], [Dem12], the discriminant $D_{27}$ of a ternary quartic is a degree 27 polynomial with coefficients in $\mathbb{Z}$ and always belongs to our HSOP (up to a multiplicative unit). It is related to the Dixmier invariant $I_{27}$ by $2^{40} \cdot I_{27}=D_{27}$. A plane quartic $F=0$ over any field is smooth if and only if $D_{27}(F) \neq 0$.

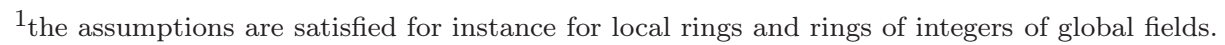


As we want to deal with valuations of the invariants, we need to introduce the following notation before stating our results.

Definition 1.5. Let $\underline{d}=\left(d_{0}, \ldots, d_{n}\right) \in \mathbb{Z}_{>0}^{n+1}$. Given an element $\underline{x}=\left(x_{0}, \ldots, x_{n}\right) \in K^{n+1} \backslash\{0\}$, we denote by $x=\left(x_{0}: \ldots: x_{n}\right)$ the corresponding point in the weighted projective space $\mathbb{P} \underline{d}(K)$. After a possible finite extension of $K$, one can always find a representative $\left(X_{0}, \ldots, X_{n}\right) \in \mathcal{O}^{n+1}$ of $x$ such that one of the $X_{i}$ 's has valuation 0 , so it represents a section in $\mathbb{P} \underline{d}(\mathcal{O})$. We call such a representative a minimal representative and denote it $\underline{x}^{\min }$

A minimal representative is not uniquely defined but two minimal representatives differ by the action of a unit and their valuations are coordinate-wise equal.

Definition 1.6. If $y \in K$ and $e \in \mathbb{Z}_{>0}$, we call the normalized valuation of degree $e$ of $y$ with respect to $\underline{x}$ the (possibly infinite) number

$$
v_{x}(y)=v(y) / e-\min \left\{v(y) / e, v\left(x_{i}\right) / d_{i}: i=0, \ldots, n\right\} \in \mathbb{Q}_{\geq 0} \cup\{\infty\},
$$

(By convention $v(0)=\infty$ ). We have $v_{x}(y)=0$ if and only if $y \neq 0$ and $x_{i}^{e} y^{-d_{i}} \in \mathcal{O}$ for all $i \leq n$.

Notice that if $\left(X_{0}, \ldots, X_{n}, Y\right)$ is a minimal representative of the point $\left(x_{0}: \ldots: x_{n}: y\right) \in \mathbb{P} \underline{d}, e$, then $v_{x}(y)=v(Y) / e$.

In the context of invariants, we will use the following definition and notation.

Definition 1.7. Let $\underline{I}=\left(I_{0}, \ldots, I_{n}\right)$ be a list of homogeneous polynomials of degree $\underline{d}$ in $K\left[T_{0}, \ldots, T_{m}\right]$. For $F \in K^{m+1} \backslash\{0\}$, if $\underline{I}(F)=\left(I_{0}(F), \ldots, I_{n}(F)\right)$ is not the zero vector, and if $J$ is a homogeneous polynomials of degree $e$ we denote by $v_{I}(J(F))$ the normalized valuation of degree $e$ of $J(F)$ with respect to $\underline{I}(F)$. It only depends on the point of $\mathbb{P} \underline{d}(K)$ defined by $F$. Finally, if $F_{0} \in \mathcal{O}^{m+1}$ and $\underline{I}\left(F_{0}\right)=\underline{I}\left(F_{0}\right)^{\text {min }}$ we say that $F_{0}$ is a minimal form.

Remark 1.8. Let $J, I_{0}, \ldots, I_{n} \in E \subseteq \mathcal{O}\left[T_{0}, \ldots, T_{m}\right]$ be homogeneous elements of some graded sub- $\mathcal{O}$ algebra and suppose that

$$
E_{+} \subseteq \sqrt{\left(I_{0}, \ldots, I_{n}\right)}
$$

that is, $\left\{I_{0}, \ldots, I_{n}\right\}$ is a homogeneous system of radical generators (HSORG) of $E$, see Definition 3.7. In particular, HSOP are HSORG with further condition on the cardinality. The condition $v_{I}(J(F))=0$ is equivalent to $F \in D_{+}(J)$, the principal open subset of Proj $E$ associated to $J$. Here $F$ is viewed as a point of $(\operatorname{Proj} E)(K)$. In particular, the condition $v_{I}(J(F))=0$ does not depend on the choice of the system $I_{0}, \ldots, I_{n}$ as long as the latter is a HSORG of $E$.

Considering ternary quartic forms $F$, a natural question, and a key argument for the sequel, is to know if, after a possible finite extension of $K$, there exists a minimal form $\mathrm{GL}_{3}(K)$-equivalent to $F$. We show in Proposition 3.13 that this is the case when $F=0$ is GIT-stable over $K$ and the list $\underline{I}$ is a HSORG over $\mathcal{O}$. Using this result, we obtain the following theorem.

Theorem 1.9 (See Theorem 3.15). Let $K$ be a discrete valuation field with valuation v, valuation ring $\mathcal{O}$ and residue field $k$ of characteristic $p \geq 0$. Let $\underline{I}$ be a list of invariants giving rise to a HSORG for ternary quartic forms under the action of $\mathrm{SL}_{3, \mathcal{O}}$. Then a smooth plane quartic $C / K: F=0$ has potentially good quartic reduction if and only if $v_{I}\left(D_{27}(F)\right)=0$ (in other words, for any invariant $I_{i}$ of degree $d_{i}$ in the list $\underline{I}$, we have $\left.I_{i}(F)^{27} / D_{27}(F)^{d_{i}} \in \mathcal{O}\right)$. 
This theorem is proved in Section 3.3. Once worked out explicit HSOP in Section 4.1, it gives a practical criterion in all characteristics. Indeed, notice, that though we use the existence of a minimal form, it is not necessary to compute it to check whether $v_{I}\left(D_{27}(F)\right)=0$. Similarly, we do not need the minimal form to compute an equation of the special fiber when the curve has potentially good quartic reduction, at least generically and if $p>7$ (see remark 4.4). Some illustrations are given in Example 4.5 and in Theorems 4.7, 4.8 and 4.9 with the characterization of potentially good quartic reduction for Picard curves in terms of low degree polynomials in their coefficients when $\operatorname{char}(K) \neq 3$.

From Section 5.1 and beyond, we restrict to a discrete valuation ring $\mathcal{O}$ with residue field $k$ of characteristic $p=0$ or $p>7$ (see a discussion about this restriction in remark 5.15). We focus there on distinguishing between curves which have potentially good hyperelliptic reduction or have not potentially good reduction. In Proposition 5.3, we first prove that $C / K: F=0$ potentially admits a (not necessarily good) toggle model if and only if any minimal representative of $\mathrm{DO}(F)$ reduces modulo $\pi$ to $\mathrm{DO}\left(Q_{0}^{2}\right)$ where $Q_{0}=x_{2}^{2}-4 x_{1} x_{3}$ is a fixed non-degenerate quadric (the choice of this particular one will become clearer later).

It remains to characterize in terms of the invariants when a toggle model $Q_{0}^{2}+\pi^{2 s} G=0$ is good, i.e. when the intersection of $\bar{Q}_{0}=0$ with $\bar{G}=0$ is transversal. This is the content of Section 5.2. In order to do so, we introduce a well-known $\mathrm{SL}_{2}(\bar{K})$-equivariant linear morphism $b_{8}$ from the space of ternary quartic forms $F$ to the space of binary octic forms. The action on the ternary quartics is given through a morphism $h: \mathrm{SL}_{2}(\bar{K}) \rightarrow \mathrm{SL}_{3}(\bar{K})$ whose image denoted $\mathrm{SO}_{3}(\bar{K})$ is the stabilizer of $Q_{0}$. Starting from a good toggle model $F=Q_{0}^{2}+\pi^{2 s} G$ of $C / K$, a quick computation shows that $b_{8}(F)=\pi^{2 s} b_{8}(G)$ and that $y^{2}=\overline{b_{8}(G)}$ is an affine equation of the special fiber of the stable model. The algebra of invariants of binary octics under the action of $\mathrm{SL}_{2}(\bar{K})$ is generated by the classical Shioda invariants $j_{2}, \ldots, j_{10}$, see [Shi67]. The invariants $j_{2}, \ldots, j_{7}$ still form a HSOP over $\mathcal{O}$, see Corollary 3.17 and the discussion before. Evaluating the Dixmier-Ohno invariants on a toggle model $Q_{0}^{2}+\pi^{2 s} G=0$ and the Shioda invariants $j_{2}, \ldots, j_{7}$ on $b_{8}(G)$ gives algebraic relations between the two sets and considering their $\pi$ expansion reveals a list $\iota=\left(\iota_{6}, \iota_{9}, \iota_{12}, \iota_{15}, \iota_{18}, \iota_{21}\right)$ of six explicit invariants for ternary quartic forms, see equation (5.3). Moreover we get a similar congruence (5.4) between the discriminant of binary octics, $D_{14}$, and $I_{3}^{5} I_{27}$. For a good toggle model, one has $v_{\iota}\left(I_{3}^{5}(F) I_{27}(F)\right)=v\left(D_{14}\left(b_{8}(G)\right)\right)=0$. Actually, we have the following equivalence.

Theorem 1.10. Let $K$ be a discrete valuation field with valuation $v$, valuation ring $\mathcal{O}$ and a uniformizer $\pi$. Let $k=\mathcal{O} /\langle\pi\rangle$ be the residue field of characteristic $p \neq 2,3,5$ and 7 . Let $C / K$ be a smooth plane quartic defined by $F=0$. Then $C$ has potentially good hyperelliptic reduction if and only if

$$
v_{\mathrm{DO}}\left(I_{3}(F)\right)=0, \quad v_{\mathrm{DO}}\left(I_{27}(F)\right)>0 \text { and } v_{\iota}\left(I_{3}(F)^{5} I_{27}(F)\right)=0 .
$$

Under these conditions, one can also obtain an explicit equation for the special fiber.

To prove that the conditions of Theorem 1.10 are also sufficient, one first observes that they imply the existence of a toggle model $F=Q_{0}^{2}+\pi^{2 s} G$ for $C$. Then, a crucial step is to prove that we can choose it such that $\overline{b_{8}(G)}$ is GIT-semi-stable. In order to do this, we rigidify a toggle model in the following way: there is, up to a multiplicative constant, a unique contravariant $\rho$ of order 2 and degree 4 , which can be seen as a quadratic form whose coefficients are degree 4 polynomials in the coefficients of $F$ (see [Dix87] and [LRS18]). After a possible finite extension of $K$ and completion of $K$, one can always find a toggle model $F$ such that $\rho(F)=\rho\left(Q_{0}^{2}\right)$. This extra-information allows us to control simultaneously the integrality of the coefficients of the toggle model and of the form $b_{8}(G)$ we are looking for (see Lemma 5.12). 
Let us point out that the conditions in Theorem 1.10 may give the wrong idea that the study of the valuations of $I_{3}$ and $I_{27}$ would be enough to characterize the reduction. But this is not true since we need to consider them inside the broader lists of invariants DO and $\iota$, and normalize them accordingly to the valuations of all the invariants of these lists. Notice that a similar phenomena occurs for Siegel modular forms. In [LR19], we show that the pull-back of the Siegel modular form $\chi_{28}$ to the space of quartics is $-2^{171} \cdot 3^{3} \cdot I_{27}^{3} I_{3}$. Now, over $\mathbb{C}$, the non-vanishing of this modular form together with the vanishing of the modular form $\chi_{18}$ (which is known to be related to $I_{27}^{2}$ ) characterizes the locus of hyperelliptic Jacobians among abelian varieties (see [Tsu86, p.847]). A natural guess is to expect that they would be the key forms to find the reduction type of a quartic. However, their expressions in terms of invariants seem to lead to the absurd statement that the vanishing of $\chi_{18}$ always implies the vanishing of $\chi_{28}$. Within this article, one sees that this is because these forms would have to be considered inside broader lists to become significant.

One of the motivations of this work was, in the continuation of [BCL $\left.{ }^{+} 15\right],\left[\mathrm{KLL}^{+} 16\right]$ and $\left[\mathrm{KLL}^{+} 18\right]$, to understand the primes dividing the discriminant $I_{27}$ of a smooth plane quartic with complex multiplication over a number field only in terms of the CM-field. We hope that the present work can be useful for this task. Another direction is to refine the study of the bad reduction type in the spirit of [BW17], [DDMM19] and to describe more precisely the degenerated cases. We only have a quick peek at this question in remarks 2.3 and 2.10 and set aside an exhaustive study for a forthcoming article.

\section{Hyperelliptic Reduction in terms of TOGgle MOdels}

In this section $K$ is a discrete valuation field with valuation $v$, valuation ring $\mathcal{O}$ and a uniformizer $\pi$. Let $k=\mathcal{O} /\langle\pi\rangle$ be the residue field of characteristic $p \geq 0$. Before proving Theorem 1.4, we need some preliminary results.

2.1. Some general facts. In this section we prove the existence of suitable models when $C$ has good hyperelliptic reduction over $K$ (see Theorems 2.8 and 2.9 ).

We start with some geometric observations. Suppose $C$ has good reduction over $K$. Let $\mathcal{C}$ be a smooth projective model over $\mathcal{O}$. Let $\omega_{\mathcal{C} / \mathcal{O}}$ be the dualizing sheaf $\left(=\Omega_{\mathcal{C} / \mathcal{O}}^{1}\right.$ as $\mathcal{C}$ is smooth $)$. Then $\omega_{\mathcal{C} / \mathcal{O}}$ is a base-point free invertible sheaf and induces a morphism

$$
f: \mathcal{C} \rightarrow \mathbb{P}\left(H^{0}\left(\mathcal{C}, \omega_{\mathcal{C} / \mathcal{O}}\right)\right) \simeq \mathbb{P}_{\mathcal{O}}^{2}
$$

On the generic fiber, $f$ is a closed immersion because $C$ is a plane quartic. Let $\mathcal{Z}=f(\mathcal{C})$ be the image of $f$ endowed with the reduced subscheme structure. Then $f: \mathcal{C} \rightarrow \mathcal{Z}$ is a birational finite morphism. As $\mathcal{C}$ is normal, $f$ is just the normalization morphism.

Fact 2.1. If we have a finite birational morphism $\mathcal{W} \rightarrow \mathcal{Z}$, and if $\mathcal{W}$ is normal, then $\mathcal{W} \rightarrow \mathcal{Z}$ is the normalization morphism ([Liu02, Proposition 4.1.22]), hence $\mathcal{W}$ is isomorphic to $\mathcal{C}$.

If $\omega_{\mathcal{C} / \mathcal{O}}$ is very ample, then $f$ is a closed immersion and $\mathcal{C}$ is a smooth quartic in $\mathbb{P}_{\mathcal{O}}^{2}$. Suppose from now on that $\omega_{\mathcal{C} / \mathcal{O}}$ is not very ample. Then $f_{k}: \mathcal{C}_{k} \rightarrow \mathbb{P}_{k}^{2}$ is a finite generically étale morphism of degree 2 into its image $\left(\mathcal{Z}_{k}\right)_{\text {red }}$ which is a smooth conic over $k$. The scheme $\mathcal{Z}$ is defined by an irreducible quartic form $F \in \mathcal{O}\left[x_{1}, x_{2}, x_{3}\right]$ as

$$
\mathcal{Z}=V_{+}(F) \subset \mathbb{P}_{\mathcal{O}}^{2}
$$

Let $q \in k\left[x_{1}, x_{2}, x_{3}\right]$ be a nondegenerate quadratic form defining $(\mathcal{Z})_{\text {red }}$ :

$$
\mathcal{Z}_{\mathrm{red}}=V_{+}(q) \subset \mathbb{P}_{k}^{2}
$$


In the sequel, $q$ will be fixed once for all. As $V_{+}(\bar{F})=\mathcal{Z}_{k}$, the reduction $\bar{F}$ is equal to a multiple of $q^{2}$. Scaling $F$ by a unit of $\mathcal{O}$, we can suppose that

$$
\bar{F}=q^{2} .
$$

Given such an $F$, equation (2.3) implies that there exist a positive integer $r>0$, a quadratic form $Q \in \mathcal{O}\left[x_{1}, x_{2}, x_{3}\right]$ and a quartic form $G \in \mathcal{O}\left[x_{1}, x_{2}, x_{3}\right]$ such that

$$
F=Q^{2}+\pi^{r} G,
$$

with $\bar{Q}=q$ and $\bar{G} \neq 0$. Note that if $\hat{\mathcal{O}}$ is the completion of $\mathcal{O}$, then $F$ is not a square in $\hat{\mathcal{O}}\left[x_{1}, x_{2}, x_{3}\right]$ because $C$ is geometrically reduced. So the biggest possible power $\pi^{r}$ dividing $F-Q^{2}$ in $\mathcal{O}\left[x_{1}, x_{2}, x_{3}\right]$ for all liftings $Q$ of $q$ exists.

We endow the polynomial ring $\mathcal{O}\left[x_{1}, x_{2}, x_{3}, y\right]$ with the grading $\operatorname{deg} x_{i}=1$ and $\operatorname{deg} y=2$. The following lemma will be repeatedly used:

Lemma 2.2. Let $B$ be a graded $\mathcal{O}$-algebra of the following form

$$
B=\mathcal{O}\left[x_{1}, x_{2}, x_{3}, y\right] /\left(Q-\pi^{s} y, y^{2}+\pi^{\varepsilon} T\right), \quad T \in \mathcal{O}\left[x_{1}, x_{2}, x_{3}\right]+\mathcal{O}\left[x_{1}, x_{2}, x_{3}\right] y
$$

with $s \geq 0, \varepsilon \in\{0,1\}$ and $2 s+\varepsilon>0$. Then the following properties hold:

(a) $B$ is flat over $\mathcal{O}$.

(b) Suppose that $B \otimes K$ is normal. If $B \otimes k$ is reduced when $\varepsilon=0$ and $T \notin(\pi, Q, y)$ when $\varepsilon=1$, then $B$ is normal.

(c) If furthermore, $F \in\left(y^{2}+\pi^{\varepsilon} T\right) K^{*}$ in $K\left[x_{1}, x_{2}, x_{3}, y\right] /\left(Q-\pi^{s} y\right)$, then $\operatorname{Proj} B \simeq \mathcal{C}$.

Proof. (a) The flatness is equivalent to $B$ being $\pi$-torsion-free. The latter follows from a straightforward computation.

(b) As $\operatorname{dim} B \otimes K=\operatorname{dim} B \otimes k=2, \operatorname{Spec} B$ is equidimensional. It is then easy to see that $B$ is a local complete intersection ([Liu02], Exercise 6.3.4(c)). By Serre's criterion ([Liu02], Corollary 8.2.24), it is then enough to check that $B_{\mathfrak{p}}$ is normal for height 1 prime ideals $\mathfrak{p}$ of $B$. This follows from the hypothesis on $B \otimes K$ if $\pi \notin \mathfrak{p}$.

Suppose that $\pi \in \mathfrak{p}$. Then $\mathfrak{p}$ is a minimal prime ideal over $\pi B$. Let us show that $B_{\mathfrak{p}}$ is a discrete valuation ring, or equivalently, that $\mathfrak{p} B_{\mathfrak{p}}$ is principal. If $\varepsilon=0$, as $B \otimes k$ is reduced, $\mathfrak{p} B_{\mathfrak{p}}$ is generated by $\pi$. If $\varepsilon=1$, then $\mathfrak{p}=(\pi, y)$ is the unique prime ideal over $\pi B$. This prime ideal is the image in $B$ of the prime ideal $(\pi, Q, y)$ of $\mathcal{O}\left[x_{1}, x_{2}, x_{3}, y\right]$. By hypothesis, $T \notin \mathfrak{p}$ in $B$, therefore $\mathfrak{p} B_{\mathfrak{p}}$ is generated by $y$.

(c) The canonical map $\mathcal{O}\left[x_{1}, x_{2}, x_{3}\right] \rightarrow B$ is finite. The hypothesis on $F$ easily implies that $F$ belongs to the ideal $\left(Q-\pi^{s} y, y^{2}+\pi^{\varepsilon} T\right)$ in $\mathcal{O}\left[x_{1}, x_{2}, x_{3}, y\right]$. So we get a canonical map $\phi: \mathcal{O}\left[x_{1}, x_{2}, x_{3}\right] /(F) \rightarrow B$ which is an isomorphism after tensoring with $K$, thus $\phi$ is finite and injective and the corresponding morphism $\operatorname{Proj} B \rightarrow \mathcal{Z}$ is finite and birational. We conclude by Fact 2.1.

Remark 2.3. When $C$ has irreducible stable (not necessarily smooth) reduction over $K$, the situation is very similar to the good reduction case. Let $\mathcal{C}$ be the stable model of $C$ over $\mathcal{O}$. Then $\omega_{\mathcal{C} / \mathcal{O}}$ is still base-point free and induces a morphism

$$
f: \mathcal{C} \rightarrow \mathbb{P}\left(H^{0}\left(\mathcal{C}, \omega_{\mathcal{C} / \mathcal{O}}\right)\right) \simeq \mathbb{P}_{\mathcal{O}}^{2}
$$

The reduced image $\mathcal{Z}=f(\mathcal{C})$ is a quartic and $f: \mathcal{C} \rightarrow \mathcal{Z}$ is a birational finite morphism, equal to the normalization morphism. If $\omega_{\mathcal{C} / \mathcal{O}}$ is very ample, then $\mathcal{C} \simeq \mathcal{Z}$ is a stable quartic in $\mathbb{P}_{\mathcal{O}}^{2}$. If $\omega_{\mathcal{C} / \mathcal{O}}$ is not very ample, then one can show that $\left(\mathcal{Z}_{k}\right)_{\text {red }}$ is a smooth conic over $k$ and $f_{k}: \mathcal{C}_{k} \rightarrow\left(\mathcal{Z}_{k}\right)_{\text {red }}$ is a finite generically étale morphism of degree 2 . The reduction is a singular hyperelliptic curve. 
Next we examine when the special fiber of $\operatorname{Proj} B$ is reduced or semi-stable (in the Deligne-Mumford sense). A point in a scheme over $k$ is said to be separable over $k$ if its residue field is a separable extension of $k$.

Lemma 2.4. Suppose char $(k) \neq 2$. Let $g \in k\left[x_{1}, x_{2}, x_{3}\right]$ be a quartic form and let

$$
W=\operatorname{Proj}\left(k\left[x_{1}, x_{2}, x_{3}, y\right] /\left(q, y^{2}+g\right)\right)
$$

with $\operatorname{deg} x_{i}=1$ and $\operatorname{deg} y=2$. Then the following properties are true:

(a) $W$ is reduced if and only if $g \neq \equiv 0 \bmod q$;

(b) $W$ is semi-stable (respectively smooth) if and only if the curves $q=0$ and $g=0$ in $\mathbb{P}_{k}^{2}$ intersect at separable points over $k$, with multiplicity at most 2 (respectively equal to 1 ).

Proof. (a) The condition is clearly necessary. Suppose that the condition is satisfied. Let us prove that $B_{k}:=k\left[x_{1}, x_{2}, x_{3}, y\right] /\left(q, y^{2}+g\right)$ is reduced. Let $A=k\left[x_{1}, x_{2}, x_{3}\right] /(q)$. This is an integrally closed domain and we have $B_{k}=A \oplus A y$. By hypothesis $g \neq 0$ in $A$. So $\operatorname{Frac}(A)[y] /\left(y^{2}+g\right)$ is $\operatorname{separable} \operatorname{over} \operatorname{Frac}(A)$. Hence,

$$
B_{k}=A \oplus A y \subset \operatorname{Frac}(A) \oplus \operatorname{Frac}(A) y=\operatorname{Frac}(A)[y] /\left(y^{2}+g\right)
$$

is reduced.

(b) The natural projection $\phi: W \rightarrow V_{+}(q)$ is finite and étale away from the subscheme $D:=V_{+}(q, g)$. Let $w_{0} \in W$ be a point lying over some point of $D$. Then $\phi$ is ramified at $w_{0}$. Let us show that $w_{0}$ is a separable point over $k$. When $W$ is smooth at $w_{0}$, this is proved for instance in [LL99], Lemma 3.3. The case when $W$ is singular at $w_{0}$ is proved in [Liu02], Proposition 10.3.7(b).

For the rest of (b), we can suppose that $k$ is algebraically closed. A local equation of $W$ at a point lying over an intersection point $z \in D$ is $y^{2}=x^{r} a$ where $r$ is the intersection number at $z$ and $a \in \mathcal{O}_{V_{+}(q), z}^{*}$. The condition for $W$ to be semi-stable or smooth is then clear.

Lemma 2.5. Let char $(k)=2$. Let $h, g \in k\left[x_{1}, x_{2}, x_{3}\right]$ be respectively a quadratic and a quartic forms. Let

$$
B_{k}=k\left[x_{1}, x_{2}, x_{3}, y\right] /\left(q, y^{2}+h y+g\right)
$$

and let $W=\operatorname{Proj} B_{k}$ with $\operatorname{deg} x_{i}=1$ and $\operatorname{deg} y=2$. Then the following properties are true:

(a) $W$ is reduced if and only if $h$ is prime to $q$ or, $h$ is divisible by $q$ and $g$ is not a square mod $q$;

(b) if $h$ is divisible by $q$, then $W$ is not semi-stable;

(c) if the curves $V_{+}(q)$ and $V_{+}(h)$ intersect transversely at separable points of $\mathbb{P}_{k}^{2}$, then $W$ is semistable.

Proof. (a) The condition is clearly necessary. Let us show the converse. Let $A$ be defined as in the proof of Lemma 2.4(a). If $h$ is prime to $q$, then the proof is similar to that of Lemma 2.4(a). Suppose now that $h \equiv 0(\bmod q)$. If $b=a_{0}+a_{1} y \neq 0$ is nilpotent in $B_{k}$, then so is $b^{2}=a_{0}^{2}+a_{1}^{2} g \in A$. Therefore $a_{0}^{2}+a_{1}^{2} g=0$ in $A$. As the latter is integrally closed, $a_{0} / a_{1} \in A$ and $g$ is a square in $A$, contradiction.

(b) Denote by $\phi: W \rightarrow Z:=V_{+}(q)$ the natural projection. If $h$ is divisible by $q$, then $\phi$ is purely inseparable, so the singularities of $W$ are all cuspidal. On the other hand, $W$ is not smooth as its arithmetic genus is 3 while its geometric genus is 0 . So $W$ is not semi-stable.

(c) We can suppose $k$ is separably closed. The above cover $W \rightarrow V_{+}(q)$ is étale away from $D$. So $W \backslash \phi^{-1}(D)$ is smooth. The local equation of $W$ at a point lying over $z \in D$ is $y^{2}+t u(t) y+g(t)$ where $t$ is a local parameter of $Z \simeq \mathbb{P}_{k}^{1}$ at $z$, with $u(0) \neq 0$. After a translation of $y$ by some constant in $\bar{k}$, the 
local equation has the shape $y^{2}+t u(t) y+t^{r} f(t)$ with $r \geq 1$ and $f(0) \neq 0$. If $r=1$, the point is smooth, otherwise it is a node.

Remark 2.6. The condition in (c) is not necessary. We can have inseparable non-transverse intersection points with $W$ smooth.

Remark 2.7. The smoothness condition is less easy to express than in the $\operatorname{char}(k) \neq 2$ case. Keep the notation of the proof of Part (b). Let $w_{0} \in W$. We know that $w_{0}$ is a smooth point if $w_{0} \notin \phi^{-1}(D)$. Suppose that $\phi\left(w_{0}\right)=z_{0} \in D$. The Jacobian criterion implies that $W$ is smooth at $w_{0}$ if and only if the matrix

$$
\left(\begin{array}{llc}
\partial q / \partial x_{1} & \partial q / \partial x_{2} & \partial q / \partial x_{3} \\
y_{0} \partial h / \partial x_{1}+\partial g / \partial x_{1} & y_{0} \partial h / \partial x_{2}+\partial g / \partial x_{2} & y_{0} \partial h / \partial x_{3}+\partial g / \partial x_{3}
\end{array}\right)
$$

where $y_{0} \in \bar{k}$ is a square root of $g\left(z_{0}\right)$, has rank 2 at $z_{0}$.

2.2. Proof of Theorem 1.4. In this subsection we prove the following result.

Theorem 2.8. Let $K$ be a discrete valuation field with valuation $v$, valuation ring $\mathcal{O}$ and a uniformizer $\pi$. Let $k=\mathcal{O} /\langle\pi\rangle$ be the residue field of characteristic $p \neq 2$. Suppose that the smooth plane quartic $C$ has good hyperelliptic reduction over K. Fix $q$ and a defining equation $F$ of $C$ as in formulas (2.2) and (2.3). Then there exist homogeneous polynomials $Q, G \in \mathcal{O}\left[x_{1}, x_{2}, x_{3}\right]$ of degrees 2,4 respectively, and a positive integer s such that

(a) $F=Q^{2}+\pi^{2 s} G$ and $\bar{G} \neq 0$;

(b) The smooth projective model $\mathcal{C}$ of $C$ over $\mathcal{O}$ is

$$
\operatorname{Proj}\left(\mathcal{O}\left[x_{1}, x_{2}, x_{3}, y\right] /\left(Q-\pi^{s} y, y^{2}+G\right)\right)
$$

in the weighted projective space $\mathbb{P}_{\mathcal{O}}^{(1,1,1,2)}$.

(c) The conic $\bar{Q}=0$ and the quartic $\bar{G}=0$ in $\mathbb{P}_{k}^{2}$ intersect transversely at an effective divisor of degree 8 over $k$;

Proof. Let $Q, G$ be as in equation (2.4) with the biggest possible $r$. We claim that $\bar{G}$ is prime to $q$. Otherwise, $G=Q R+\pi G_{1}$ with $R, G_{1}$ homogeneous of degrees 2 and 4 respectively. Then the polynomial $F-\left(Q+\pi^{r} R / 2\right)^{2}$ is divisible by $\pi^{r+1}$. Contradiction with the assumption on $r$.

We now prove the theorem. Let $s=\lfloor r / 2\rfloor$ and $\varepsilon=r-2 s \in\{0,1\}$. Let

$$
\mathcal{W}=\operatorname{Proj}\left(\mathcal{O}\left[x_{1}, x_{2}, x_{3}, y\right] /\left(Q-\pi^{s} y, y^{2}+\pi^{\varepsilon} G\right)\right) .
$$

If $\varepsilon=1$, as $G \notin(\pi, Q, y)$, Lemma 2.2 implies that $\mathcal{W}$ is normal and isomorphic to $\mathcal{C}$, hence smooth. This is absurd as the special fiber of $\mathcal{W}$ is non-reduced. So $\varepsilon=0$. The special fiber $\mathcal{W}_{k}$ is reduced by Lemma 2.4 (a). Again by Lemma $2.2(\mathrm{c}), \mathcal{W} \simeq \mathcal{C}$. Part (c) is just Lemma 2.4(b).

2.3. Good hyperelliptic reduction in $\operatorname{char}(k)=2$. When the characteristic of $k$ is equal to 2 , toggle models have singular close fibers. However, we still have an analog result to Theorem 1.4 in this case.

Theorem 2.9. Let $K$ be a discrete valuation field with valuation $v$, valuation ring $\mathcal{O}$ and a uniformizer $\pi$. Let $k=\mathcal{O} /\langle\pi\rangle$ be the residue field of characteristic $p=2$. Suppose that the smooth plane quartic $C$ has good hyperelliptic reduction over $K$. Fix $q$ and a definition equation $F$ of $C$ as in formulas (2.2) and (2.3). Then there exist homogeneous polynomials

$$
Q, H, G \in \mathcal{O}\left[x_{1}, x_{2}, x_{3}\right]
$$

of degrees 2, 2, 4 respectively, $u \in 1+\pi \mathcal{O}$ and a positive integer s such that 
(a) $u F=Q^{2}+\pi^{s} Q H+\pi^{2 s} G$ and $\bar{H}, \bar{G}$ are prime to $q$;

(b) The smooth projective model $\mathcal{C}$ of $C$ over $\mathcal{O}$ is

$$
\operatorname{Proj}\left(\mathcal{O}\left[x_{1}, x_{2}, x_{3}, y\right] /\left(Q-\pi^{s} y, y^{2}+H y+G\right)\right)
$$

in the weighted projective space $\mathbb{P}_{\mathcal{O}}^{(1,1,1,2)}$.

Proof. In all the proof, capitalized italic letters denote homogeneous polynomials in $\mathcal{O}\left[x_{1}, x_{2}, x_{3}\right]$ of degree 2 or 4 (the exact degree will be clear from the context).

Step 1. Suppose we have a decomposition

$$
F=Q^{2}+\pi^{s} Q H+\pi^{r} P
$$

with $1 \leq r<2 s$ (including $s=+\infty$, in which case $\pi^{s}$ stands for 0 ). Then we have a new decomposition

$$
F=Q_{1}^{2}+\pi^{s_{1}} Q_{1} H_{1}+\pi^{r+1} P_{1}
$$

with $Q_{1} \in Q+\pi^{[r / 2]} \mathcal{O}\left[x_{1}, x_{2}, x_{3}\right]$ and $s_{1} \geq \min \{s, r, v(2)+r / 2\}$, where $v(2)$ is the normalized $K$-valuation of 2 .

Let $\ell=[r / 2]$ and $\varepsilon=r-2 \ell$. Consider

$$
\mathcal{W}=\operatorname{Proj}\left(\mathcal{O}\left[x_{1}, x_{2}, x_{3}, y\right] /\left(Q-\pi^{\ell} y, y^{2}+\pi^{s-\ell} H y+\pi^{\varepsilon} P\right)\right) .
$$

Case 1. Suppose that $\varepsilon=1(\ell \geq 0)$. The special fiber of $\mathcal{W}$ is not reduced (Lemma 2.5(a) if $\ell>0)$, by Lemma 2.2 (b) and (c), we must have

$$
P \in\left(\pi, Q_{0}, y\right) \cap \mathcal{O}\left[x_{1}, x_{2}, x_{3}\right]=(\pi, Q) \mathcal{O}\left[x_{1}, x_{2}, x_{3}\right] .
$$

Write $P=Q H_{0}+\pi P_{1}$, then

$$
F=Q^{2}+\left(\pi^{s} H+\pi^{r} H_{0}\right) Q+\pi^{r+1} P_{1} .
$$

Case 2. Suppose that $\varepsilon=0$. As $s>\ell, \mathcal{W}_{k}$ is not semi-stable by Lemma 2.5(b). Similarly to Case 1 , this implies that $\mathcal{W}_{k}$ is not reduced and $\bar{P}$ is a square $\bmod q$. Write $P=R^{2}+Q H_{0}+\pi P_{0}$. Let $Q_{1}=Q+\pi^{\ell} R$. Then

$$
F=Q_{1}^{2}+\left(\pi^{s} H+\pi^{r} H_{0}-2 \pi^{\ell} R\right) Q_{1}+\pi^{r+1} P_{1} .
$$

This achieves Step 1.

Step 2. The polynomial $F$ admits a decomposition

$$
u F=Q^{2}+\pi^{s} Q H+\pi^{2 s} G
$$

with $\bar{H}$ prime to $q$ and $u \in 1+\pi \mathcal{O}$.

Let us first show that $F$ admits a decomposition

$$
F=Q^{2}+\pi^{s} Q H+\pi^{2 s} G .
$$

Suppose that this is not true. Starting with a relation (2.4) and using repeatedly Step 1, we construct sequences $Q_{n}, P_{n}, H_{n} \in \mathcal{O}\left[x_{1}, x_{2}, x_{3}\right]$ and $s_{n} \in \mathbb{N}^{*} \cup\{+\infty\}$ such that

$$
F=Q_{n}^{2}+\pi^{s_{n}} Q_{n} H_{n}+\pi^{r+n} P_{n}
$$

and

$$
Q_{n+1}-Q_{n} \in \pi^{[(r+n) / 2]} \mathcal{O}\left[x_{1}, x_{2}, x_{3}\right], s_{n} \geq \min \left\{s_{1}, r, v(2)+r / 2\right\} \text { for all } n \geq 1 .
$$

The sequence $Q_{n}$ admits a limit $\hat{Q} \in \hat{\mathcal{O}}\left[x_{1}, x_{2}, x_{3}\right]$. As $\hat{Q}$ reduces $\bmod \pi$ to $q \neq 0$, this implies that $Q_{n}+\pi^{s_{n}} H_{n}$ converges to some $\hat{R} \in \hat{\mathcal{O}}\left[x_{1}, x_{2}, x_{3}\right]$ such that $F=\hat{Q} \hat{R}$. Contradiction because $C$ is geometrically integral. 
Now we prove the assertion of Step 2. Start with a relation (2.5). If $\bar{H}$ is prime to $q$ then we are done. Otherwise, write $H=b Q+\pi H_{0}$ with $b \in \mathcal{O}$. Then

$$
F=\left(1+b \pi^{s}\right) Q^{2}+\pi^{s+1} Q H_{0}+\pi^{2 s} G .
$$

Similarly to the above, applying repeatedly Step 1 to $\left(1+b \pi^{s}\right)^{-1} F$, we find

$$
\left(1+b \pi^{s}\right)^{-1} F=Q_{1}^{2}+\pi^{s_{1}} Q_{1} H_{1}+\pi^{2 s_{1}} G_{1}
$$

with $s_{1} \geq s+1, Q_{1}-Q \in \pi^{s} \mathcal{O}\left[x_{1}, x_{2}, x_{3}\right]$. If $\bar{H}_{1}$ is prime to $q$, then we are done. Otherwise there exist $s_{2} \geq s_{1}+1, b_{1} \in \mathcal{O}$ such that

$$
\left(\left(1+b \pi^{s}\right)\left(1+b_{1} \pi^{s_{1}}\right)\right)^{-1} F=Q_{2}^{2}+\pi^{s_{2}} Q_{2} H_{2}+\pi^{2 s_{2}} G_{2} .
$$

Again by the geometric integrality of $C$, we see that this process must stop after finitely many steps.

Step 3 Consider a relation given by Step 2. Let

$$
\mathcal{W}=\operatorname{Proj}\left(\mathcal{O}\left[x_{1}, x_{2}, x_{3}, y\right] /\left(Q-\pi^{s} y, y^{2}+H y+G\right)\right) .
$$

As $q$ is prime to $\bar{H}, \mathcal{W}_{k}$ is reduced by Lemma 2.5 (a), hence $\mathcal{W} \simeq \mathcal{C}$ by Lemma 2.2 (b) and (c). This implies that $\bar{G}$ is prime to $q$ because $\mathcal{C}_{k}$ is irreducible.

Remark 2.10. Suppose that $C$ has stable irreducible reduction over $K$. Then Theorems 2.8 and 2.9 hold after replacing smooth by stable, and in $2.8(\mathrm{c}), V_{+}(q)$ and $V_{+}(\bar{G})$ intersect with multiplicities at most 2 instead of 1 . The proof is exactly the same by using Remark 2.3. For the general case, there are several dozens of stable reduction types for curves of genus 3. At the present moment it seems difficult to find characterizations for all types in a similar way as above. However, we think that some types can be dealed with the techniques we develop in this work. This will be the purpose of a future article.

Remark 2.11. Let $\mathcal{O}$ be a PID with field of fractions $K$ and $C: F=0$ be a smooth projective plane quartic with good reduction everywhere over $K$. Gathering the local information above, one can give a smooth model of $C$ over $\mathcal{O}$ by

$$
\mathcal{C}=\operatorname{Proj}\left(\mathcal{O}\left[x_{1}, x_{2}, x_{3}, y\right] /\left(Q-\delta y, u y^{2}+H y+G\right)\right)
$$

where $Q, H, G \in \mathcal{O}\left[x_{1}, x_{2}, x_{3}\right]$ are of degrees $2,2,4, \delta \in \mathcal{O}$ is divisible by a prime $\mathfrak{p}$ if and only if $C$ has hyperelliptic reduction at $\mathfrak{p}$ and $u \in \mathcal{O}$ is congruent to 1 modulo the primes for which $C$ has hyperelliptic reduction.

\section{Geometric Invariant Theory and characterization of potentially good quartic} REDUCTION

This section starts by gathering some definitions and results on the geometric invariant theory over rings. The main reference is [Ses77]. We derive Corollary 3.5 which unifies similar results present in the literature under more restrictive hypotheses. When $\boldsymbol{X}=\mathbb{P}^{14}$, the space of plane quartics (represented by their 15 coefficients), under the action of $G=\mathrm{SL}_{3, \mathbb{Z}}$, this corollary says the following: given a discrete valuation ring $\mathcal{O}$ with field of fractions $K$ and a smooth plane quartic $\mathrm{x} \in \boldsymbol{X}(K)$, after a possible finite extension of $K$, we can find an integral model $\mathfrak{x} \in X(\mathcal{O})$ such that its reduction is GIT-semi-stable (whereas the reduction of a naive model of $x$ may be GIT-unstable).

We then need a second ingredient. Over an algebraically closed field, a Homogeneous System Of Parameters (HSOP, see Definition 3.7) is a "minimal" set of invariants which vanish exactly on the GIT-unstable locus. We show in Section 3.2 that for a class of nice rings $R$, there exists such a set of 
invariants defined over $R$ which keep this property over the algebraic closures of all residue fields of $R$ simultaneously. Remarks 3.8, 3.9 and 3.10 will then help when working out such HSOP explicitly in Section 4.1.

Finally, in Section 3.3, we apply these results to the characterization of the reduction type. Roughly speaking, the model $\mathfrak{X}$ is a minimal form (Definition 1.7) since we know that one of the value of an invariant in the HSOP is not zero on the special fiber. Hence its invariants are a minimal representative of the invariants of $x$ (Definition 1.5). Moreover, the valuations of this representative can easily be computed from the values of the invariants without knowing $\mathfrak{X}$. Looking at the valuation of the discriminant with respect to the HSOP (Definition 1.6) will then give us the reduction type of $\mathfrak{x}$ and therefore the type of the potential reduction of $x$.

3.1. Some results on Geometry Invariant Theory (GIT). We let $S=\operatorname{Spec} R$ be a noetherian affine scheme, and $G$ be an affine smooth group scheme over $S$ with connected and reductive geometric fibers (for instance $\mathrm{SL}_{n, R}, \mathrm{GL}_{n, R}$ ). Let $V$ be a finite rank free $R$-module endowed with an action of $G$ :

$$
G \rightarrow \operatorname{Aut}_{R}(V)
$$

(morphism of group schemes). Then $G$ acts "linearly" on the projective space $\mathbb{P}\left(V^{\vee}\right)$, where $V^{\vee}$ is the dual of $V$. Let $\boldsymbol{X}$ be a $G$-stable closed subscheme of $\mathbb{P}\left(V^{\vee}\right)$ of the form $\boldsymbol{X}=\operatorname{Proj} B$ where $B$ is a $G$-stable homogeneous quotient of the symmetric algebra $\operatorname{Sym}\left(V^{\vee}\right)$ over $R$. The $\operatorname{ring}$ of $G$-invariants $B^{G}$ is

$$
B^{G}=\left\{b \in B \mid \sigma\left(b \otimes 1_{A}\right)=b \otimes 1_{A}\right\}
$$

where $A$ runs through the $R$-algebras and $\sigma \in G(A)$. When $R$ is an algebraically closed field $k$, the invariants of $B$ can be found using only the $k$-rational points of $G$ and hence it corresponds to the usual definition. Namely, denote by $B^{G(k)}=\{b \in B \mid g \cdot b=b, \forall g \in G(k)\} \supseteq B^{G}$, we have the following result.

Proposition 3.1. Let $k$ be an algebraically closed field and let $B$ be a $k$-algebra endowed with the action of a smooth affine algebraic group $G$. Then $B^{G}=B^{G(k)}$.

Proof. Let $b \in B^{G(k)}$. Let $A$ be a $k$-algebra. We have to show that $b \otimes 1_{A} \in B \otimes_{k} A$ is invariant by all $\sigma \in G(A)$. Let $h:=\sigma\left(b \otimes 1_{A}\right)-b \otimes 1_{A} \in B \otimes_{k} A$.

First suppose that $A$ is reduced and of finite type over $k$. For any closed point $y \in \operatorname{Spec} A$ the image of $h$ by the canonical homomorphism

$$
B \otimes_{k} A \rightarrow B \otimes_{k} k(y)=B \otimes_{k} k=B
$$

is $\sigma(y)\left(b \otimes 1_{k(y)}\right)-b \otimes 1_{k(y)}=\sigma(y)(b)-b=0$, where $\sigma(y) \in G(k(y))=G(k)$ is the image of $\sigma$ by $G(A) \rightarrow G(k(y))$. Let $\left\{e_{i}\right\}_{i}$ be a basis of $B$ as $k$-vector space. Then $h=\sum_{i} e_{i} \otimes a_{i}$ with $a_{i} \in A$ and we have $a_{i}(y)=0$ for all closed points $y \in \operatorname{Spec} A$. Hence $a_{i}=0$ and $h=0$.

Now we consider the general case. Let $A$ be any $k$-algebra and let $\sigma \in G(A)$. Let us denote by $\sigma^{\#}: A_{0}:=\mathcal{O}_{G}(G) \rightarrow A$ the corresponding $k$-algebra homomorphism. Let $\sigma_{0} \in G\left(A_{0}\right)$ be the identity morphism. Then the map $G\left(A_{0}\right) \rightarrow G(A)$ induced by $\sigma^{\#}$ takes $\sigma_{0}$ to $\sigma$ and $h$ is the image of the element $\sigma_{0}\left(b \otimes 1_{A_{0}}\right)-b \otimes 1_{A_{0}}$ by $\operatorname{Id}_{B} \otimes \sigma^{\#}$. Applying the previous case to the reduced $k$-algebra of finite type $A_{0}$, we get $h=0$.

Corollary 3.2. Let $R$ be an integral domain with field of fractions $K$ and let $\bar{K}$ be an algebraic closure of $K$. Let $B$ be a flat $R$-algebra endowed with the action of a smooth affine algebraic group $G$ over $R$. Then

$$
B^{G}=\left\{b \in B \mid b \otimes 1_{\bar{K}} \in\left(B \otimes_{R} \bar{K}\right)^{G(\bar{K})}\right\}
$$


Proof. Let $b \in B$. To check that $b \in B^{G}$, as in the proof of Proposition 3.1, it is enough to check that $\sigma\left(b \otimes 1_{A_{0}}\right)=b \otimes 1_{A_{0}}$ in $B \otimes_{R} A_{0}$ where $A_{0}=\mathcal{O}_{G}(G)$. As $B$ and $A_{0}$ are flat over $R$, the morphism $B \otimes_{R} A_{0} \rightarrow\left(B \otimes_{R} \bar{K}\right) \otimes_{\bar{K}}\left(A_{0} \otimes_{R} \bar{K}\right)$ is injective. So it is enough to check the desired equality over $\bar{K}$. This then follows from Proposition 3.1.

Let $R=k$ be an algebraically closed field. A point $\mathrm{x} \in \boldsymbol{X}(k)$ is GIT-semi-stable if there is a rational point $\hat{x}$ in the affine cone $\hat{\boldsymbol{X}}$ of $\boldsymbol{X}$ lying over $\times$ such that $0 \notin \overline{G(k) \cdot \hat{x}}$ (Zariski closure in $\hat{\boldsymbol{X}}$ ). It is GITstable if $G(k) \cdot \hat{\mathrm{x}}$ is closed of $\operatorname{dimension} \operatorname{dim} G$ (i.e. the stabilizer of $G$ at $\hat{x}$ is finite). When $\operatorname{dim} G>0$, GIT-stable points are also GIT-semi-stable.

For arbitrary $R$, a geometric point $\mathrm{x} \in \boldsymbol{X}(k)$, where $k$ an $R$-algebra which is an algebraically closed field, is GIT-semi-stable (resp. GIT-stable) if it is GIT-semi-stable (resp. GIT-stable) for the action of $G \times{ }_{S}$ Spec $k$ on $\boldsymbol{X} \times{ }_{S}$ Spec $k$. Then $\times$ is GIT-semi-stable if and only if there exists a homogeneous $f \in B^{G}$ of positive degree such that $\mathrm{x} \in D_{+}(f)$, see [Ses77, Thm. 1]. As a consequence, the geometric points of the open subset $\boldsymbol{X}^{\mathrm{ss}}:=\cup_{f} D_{+}(f)$ of $\boldsymbol{X}$, where $f$ runs through the homogeneous elements of $B^{G}$ of positive degrees, are exactly the GIT-semi-stable geometric points of $\boldsymbol{X}$.

Theorem 3.3 ([Ses77], Theorem 4, p. 269 and Remarks 8, 9, p. 269, 271). Let $R$ be a noetherian ring. Then we have the following properties.

(i) The inclusion $B^{G} \subseteq B$ induces an affine surjective morphism

$$
\phi: \boldsymbol{X}^{\mathrm{ss}} \rightarrow \boldsymbol{Y}:=\operatorname{Proj}\left(B^{G}\right) .
$$

This morphism is a categorical quotient: any G-invariant morphism $\boldsymbol{X}^{\mathrm{ss}} \rightarrow \boldsymbol{Z}$ with trivial action on $\boldsymbol{Z}$ factors uniquely through $\boldsymbol{Y} \rightarrow \boldsymbol{Z}$. The formation of this quotient commutes with flat base changes $R \rightarrow R^{\prime}$.

(ii) If $\boldsymbol{Z}$ is a $G$-stable closed subscheme of $\boldsymbol{X}$, then $\phi(\boldsymbol{Z})$ is closed in $\boldsymbol{Y}$.

(iii) For any pair of geometric points $\mathrm{x}_{1}, \mathrm{x}_{2} \in \boldsymbol{X}^{\mathrm{ss}}(k)$, we have $\phi\left(\mathrm{x}_{1}\right)=\phi\left(\mathrm{x}_{2}\right)$ if and only if

$$
\overline{G(k) \cdot \mathrm{x}_{1}} \cap \overline{G(k) \cdot \mathrm{x}_{2}} \neq \emptyset \text {. }
$$

(iv) There is an open subset $\boldsymbol{Y}^{s} \subseteq \boldsymbol{Y}$ such that the geometric points of $\boldsymbol{X}^{\mathrm{s}}:=\phi^{-1}\left(\boldsymbol{Y}^{s}\right)$ are exactly the GIT-stable points. In particular, if $\mathrm{x}_{1}, \mathrm{x}_{2} \in \boldsymbol{X}^{s}(k)$ are two geometric points, then $\phi\left(\mathrm{x}_{1}\right)=\phi\left(\mathrm{x}_{2}\right)$ if and only if $\mathrm{x}_{2} \in G(k) \cdot \mathrm{x}_{1}$.

(v) If $R$ is an excellent ring ${ }^{2}$, then $B^{G}$ is of finite type over $R$ and $\boldsymbol{Y}$ is projective over $R$.

Remark 3.4. In [Bur92, Appendice], some clarifications are added to the proof of [Ses77, Prop.8].

The categorical quotient $\boldsymbol{Y}$ is denoted by $\boldsymbol{X}^{\mathrm{ss}} / / G$. Let $\mathrm{y} \in\left(\boldsymbol{X}^{\mathrm{ss}} / / G\right)(k)$ be a geometric point. Then $\phi^{-1}(\mathrm{y})$ is a union of closures of orbits. Any orbit of smallest dimension contained in $\phi^{-1}(\mathrm{y})$ is closed, hence contained in (and equal to) the intersection of all these closures. It is a minimal orbit.

The following result appears in [Sha80, Prop.2.1] and [Mum77, Lem.5.3] in equal characteristics setting, in [Bur92, p.122] over the $p$-adics for $\boldsymbol{X}=\mathbb{P}(V)$ and in [Sil98] and [STW14] in a dynamical context.

Corollary 3.5. Let $R=\mathcal{O}$ be a discrete valuation ring with field of fractions $K$ and such that the categorical quotient $\boldsymbol{X}^{\mathrm{ss}} / / G$ is of finite type (hence projective) over $\mathcal{O}$. Let $\mathrm{x} \in \boldsymbol{X}^{s}(K)$. Then there exists an extension $\mathcal{O} \subseteq \mathcal{O}^{\prime}$ of discrete valuation rings with $K^{\prime}=\operatorname{Frac}\left(\mathcal{O}^{\prime}\right)$ finite over $K$, and an integral point

\footnotetext{
${ }^{2}$ See [EGA], IV 7.8.2. Localizations of algebras of finite type over a field, Dedekind domains of characteristic 0, complete noetherian local rings are excellent ([EGA], IV.7.8.3(ii)-(iii)). Excellent rings are universally Japanese ([EGA], IV.7.8.3(vi)).
} 
$\mathfrak{X} \in \boldsymbol{X}^{\mathrm{ss}}\left(\mathcal{O}^{\prime}\right)$ such that $\mathfrak{X}_{K^{\prime}} \in G\left(K^{\prime}\right) \cdot \mathbf{x}$. Moreover, we can ask the image of the closed point of $\mathfrak{X}$ to belong to a minimal orbit.

Proof. Let $\mathrm{y}=\phi(\mathrm{x}) \in \boldsymbol{Y}^{s}(K)$. Let $\Gamma=\overline{\{\mathrm{y}\}} \subseteq \boldsymbol{Y}$. As $\boldsymbol{Y} / S$ is projective, $\Gamma \rightarrow S$ is an isomorphism. Let $W_{K}$ be the closed subset $\phi^{-1}(\Gamma)_{K}=\phi^{-1}(\mathrm{y})$ of $\boldsymbol{X}_{K}^{\text {ss }}$ endowed with the reduced subscheme structure. Let $W=\overline{\phi^{-1}(\Gamma)_{K}} \subseteq \boldsymbol{X}^{\mathrm{ss}}$ be its scheme-theoretical closure. As $W_{K}$ is a homogeneous space under $G_{K}$, hence irreducible, then so is $W$. Therefore $W$ is an integral scheme dominating $S=\operatorname{Spec} \mathcal{O}$, hence flat over $S$. It is a $G$-stable closed subscheme of $\boldsymbol{X}^{\mathrm{ss}}$. By Theorem 3.3(ii), $\phi(W) \subseteq \Gamma$ is closed hence equal to $\Gamma$. So $W \rightarrow S$ is surjective. By [Liu02], Prop. 10.1.36, any closed point $w$ of the (non-empty) special fiber of $W \rightarrow S$ belongs to an irreducible closed subscheme $\Gamma^{\prime} \subseteq W$ quasi-finite and surjective over $S$.

Let $\operatorname{Spec} \mathcal{O}^{\prime}$ be the localization at a closed point of the normalization of $\Gamma^{\prime}$. Note that $\mathcal{O}^{\prime}$ is a discrete valuation ring. The canonical morphism $\mathfrak{x}: \operatorname{Spec} \mathcal{O}^{\prime} \rightarrow \boldsymbol{X}^{\mathrm{ss}}$ is an integral point as we are looking for: its generic point belongs to the same geometric orbit as $\mathrm{x}$, so $\mathfrak{x}_{K^{\prime}} \in G\left(K^{\prime}\right)$.x after a finite extension of $K^{\prime}$ if necessary. Moreover, the closure of the orbit of $w$ is contained in the closed fiber $W_{s}$, so the minimal orbit is also contained in $W_{s}$. Therefore we can chose $w$ in the minimal orbit.

Now let us say a few words on the fibers of $\boldsymbol{X}^{\mathrm{ss}} / / G \rightarrow S$. Let $G, \boldsymbol{X}$ and $S=\operatorname{Spec} R$ be as in Theorem 3.3. For any $T \rightarrow S$ with $T$ affine noetherian, we have $\left(\boldsymbol{X} \times_{S} T\right)^{\mathrm{ss}}=\boldsymbol{X}^{\mathrm{ss}} \times_{S} T$. By the categorical quotient property, we have a canonical morphism

$$
\left(\boldsymbol{X}^{\mathrm{ss}} \times{ }_{S} T\right) / / G_{T} \rightarrow\left(\boldsymbol{X}^{\mathrm{ss}} / / G\right) \times{ }_{S} T .
$$

This is an isomorphism if $T \rightarrow S$ is flat [Ses77, p. 271]. In general, this morphism induces a bijection on the geometric points by Theorem 3.3(iii). If both sides are projective schemes over $T$ (e.g. $S, T$ are both excellent), then the morphism is projective [Liu02, Corollary 3.3.32(e)], hence finite [Liu02, Corollary 4.4.7] and bijective. In particular, if $R$ is excellent, then for any geometric point $s \in S(k)$ we get a finite bijective, and hence homeomorphic, morphism of projective schemes over $k$ :

$$
\left(\boldsymbol{X}_{s}\right)^{\mathrm{ss}} / / G_{s} \rightarrow\left(\boldsymbol{X}^{\mathrm{ss}} / / G\right)_{s}
$$

Remark 3.6. Suppose that $\boldsymbol{X}, G$ and its action on $\boldsymbol{X}$ are all defined over $\mathbb{Z}$. Then for any integral domain $A$, the categorical quotient $\left(\boldsymbol{X}_{A}\right)^{\mathrm{ss}} / / G_{A}$ is projective over $A$. Indeed, if $A$ has characteristic zero, then it is flat over $\mathbb{Z}$ and $\left(\boldsymbol{X}_{A}\right)^{\mathrm{ss}} / / G_{A}$ is obtained by base change from $\boldsymbol{X}^{\mathrm{ss}} / / G$ which is projective thanks to Theorem $3.3(\mathrm{v})$. Otherwise $A$ contains a prime field $\mathbb{F}_{p}$, and $\left(\boldsymbol{X}_{A}\right)^{\mathrm{ss}} / / G_{A}$ is obtained by the (flat) base change from $\left(\boldsymbol{X}_{\mathbb{F}_{p}}\right)^{\text {ss }} / / G_{\mathbb{F}_{p}}$ to $A$.

\subsection{Homogeneous system of parameters.}

Definition 3.7. Let $E$ be a graded algebra over a ring $R$.

(i) A family of homogeneous elements $f_{0}, f_{1}, \ldots, f_{m} \in E_{+}$is called a Homogeneous System Of Radical Generators (HSORG) over $R$ if $V_{+}\left(f_{0}, \ldots, f_{m}\right)=\emptyset$ in Proj $E$. This is equivalent to the inclusion $E_{+} \subseteq \sqrt{\left(f_{0}, \ldots, f_{m}\right)}$, where $E_{+}$is the irrelevant ideal of $E$.

(ii) If the fibers of $\operatorname{Proj} E \rightarrow \operatorname{Spec} R$ all have the same dimension $d \geq 0$, a Homogeneous System Of Parameters (HSOP) over $R$ of $E$ consists in a HSORG with $d+1$ elements. Note that $d+1$ is the smallest possible cardinality of a HSORG.

Remark 3.8. Let $A$ be an $R$-algebra. Any HSORG $\left\{f_{0}, \ldots, f_{m}\right\}$ of $E$ gives rise to a HSORG $\left\{f_{0} \otimes\right.$ $\left.1, \ldots, f_{m} \otimes 1\right\}$ of $E \otimes_{R} A$. The converse is true if $R \rightarrow A$ is faithfully flat (e.g. a field extension). Under the condition (ii) of the definition, the fibers of the morphism $\operatorname{Proj}\left(E \otimes_{R} A\right) \rightarrow \operatorname{Proj} A$ have $\operatorname{dimension} d$ 
and $\left\{f_{0} \otimes 1, \ldots, f_{d} \otimes 1\right\}$ is a HSOP of $E \otimes_{R} A$. Conversely, for $f_{0}, \ldots, f_{m}$ to be a HSORG (resp. HSOP) of $E$, it is enough that their images in $E \otimes_{R} k(t)$ form a HSORG (resp. HSOP) for all points $t \in \operatorname{Spec} R$. If $R=\mathcal{O}$ is a discrete valuation ring, by the properness of $\operatorname{Proj} E \rightarrow \operatorname{Spec} R$, a list of homogeneous elements of $E$ is a HSORG (resp. HSOP) over $\mathcal{O}$ if and only if their images in $E \otimes_{\mathcal{O}} k$ form a HSORG (resp. HSOP) over the algebraic closure of the residue field $k$.

Remark 3.9. Let $B$ be a homogeneous algebra over a field $k$, let $G$ be a reductive algebraic group over $k$ acting on $B$. Let $f_{0}, \ldots, f_{d} \in B^{G}=E$ be homogeneous with $d=\operatorname{dim} \operatorname{Proj} E$. Then they form a HSOP over $k$ of $E$ if in $\operatorname{Spec} B$, we have $V\left(f_{0}, \ldots, f_{d}\right)=V(\mathfrak{m} B)$ (the null-cone) where $\mathfrak{m}=E_{+}$. Moreover $E$ will be a finite algebra over $k\left[f_{0}, \ldots, f_{d}\right]$, see [DK02, Lemma 2.4.5.].

Remark 3.10. Let $R, B, G$ be as in the beginning of this section. Let $A$ be a noetherian $R$-algebra.

(i) Any HSORG of $B^{G}$ generates a HSORG of $\left(B \otimes_{R} A\right)^{G_{A}}$ by the surjectivity of the morphism (3.1).

(ii) If moreover $B^{G}$ and $\left(B \otimes_{R} A\right)^{G_{A}}$ are of finite type respectively over $R$ and $A$, then the same statement holds for any HSOP of $B^{G}$ because for any $t \in \operatorname{Spec} A$ lying over some $s \in S$, $\left(\operatorname{Proj}\left(B \otimes_{R} A\right)^{G_{A}}\right)_{k(t)}$ is homeomorphic to $\left(\operatorname{Proj} B^{G}\right)_{s} \times_{\operatorname{Spec} k(s)} \operatorname{Spec} k(t)$, hence they have the same dimension.

(iii) Let $R=\mathcal{O}$ be a discrete valuation ring with residue field $k$, and suppose $B^{G}$ is of finite type over $\mathcal{O}$. Using Proposition 3.1 and the above remarks, we see that a list of homogeneous elements of $B^{G}$ is a HSOP of $B^{G}$ if and only if their images in $B \otimes_{\mathcal{O}} \bar{k}$ define the zero set $V\left(\left(B \otimes_{\mathcal{O}} \bar{k}\right)_{+}^{G(\bar{k})}\left(B \otimes_{\mathcal{O}} \bar{k}\right)\right)$ or equivalently they form a HSOP of $\left(B \otimes_{\mathcal{O}} \bar{k}\right)^{G(\bar{k})}$ over $\bar{k}$. We can then use classical methods of GIT over an algebraically closed field to prove that a given list of homogeneous elements of $B^{G}$ is a HSOP of $B^{G}$.

A natural question is when a HSOP exists. A pictorsion ring ([GLL15], Definition 0.3) is a commutative ring $R$ such that for any finite homomorphism $R \rightarrow A$, the group $\operatorname{Pic}(A)$ is of torsion. The ring of integers of a finite extension of $\mathbb{Q}$, the ring of regular functions of an affine connected regular curve over a finite field, and semi-local rings are pictorsion.

Proposition 3.11. Let $R$ be a noetherian pictorsion ring. Let $E$ be a homogeneous $R$-algebra such that the fibers of $\operatorname{Proj} E \rightarrow \operatorname{Spec} R$ all have the same dimension $d \geq 0$. Then $E$ has a HSOP over $R$.

Proof. This is done in the proof of Theorem 8.1 in [GLL15].

Now we return to the early situation of Section 3.1 where $\boldsymbol{X}=\operatorname{Proj} B$ has linear $G$-action.

Corollary 3.12. Suppose that $R$ is excellent, pictorsion, and $S=\operatorname{Spec} R$ is connected. Assume moreover that $\boldsymbol{X}$ is flat over $S$, that $\boldsymbol{X}_{s}$ is irreducible and has a GIT-stable point for all $s \in S$. Then $B^{G}$ admits a HSOP over R.

Proof. All fibers of $\boldsymbol{X} / S$ (resp. of $G / S$ ) have the same dimension $n$ (resp. $m$ ). For all $s \in S$, as $\boldsymbol{X}_{s}$ is irreducible, its non-empty open subsets $\left(\boldsymbol{X}_{s}\right)^{\text {ss }}$ and $\left(\boldsymbol{X}_{s}\right)^{\mathrm{s}}$ are irreducible of dimension $n$. Therefore $\left(\boldsymbol{X}_{s}\right)^{\mathrm{s}} / / G_{s}$ has $\operatorname{dimension} \operatorname{dim}\left(\boldsymbol{X}_{s}\right)^{\mathrm{s}}-\operatorname{dim} G_{s}=n-m$. Let $\boldsymbol{Y}=\boldsymbol{X}^{\mathrm{ss}} / / G=\operatorname{Proj}\left(B^{G}\right)$. For all $s \in S$, the fiber $\boldsymbol{Y}_{s}$, which is homeomorphic to $\left(\boldsymbol{X}_{s}\right)^{\text {ss }} / / G_{s}$, is irreducible and contains an open subset of dimension $n-m$. So $\operatorname{dim} \boldsymbol{Y}_{s}=n-m$ for all $s \in S$. Now we can apply Proposition 3.11. 
3.3. Characterization of potentially good quartic reduction. Let $R=\mathcal{O}$ be a discrete valuation ring with field of fractions $K$ and $\boldsymbol{X}=\operatorname{Proj} B$ such that $B^{G}$ is of finite type over $\mathcal{O}$. This last property implies that $B^{G}$ admits a HSORG $\underline{I}$. By definition, a geometric point $\mathrm{x} \in \boldsymbol{X}(k)$ is GIT-unstable (i.e. not GIT-semi-stable) if and only if $\mathrm{x} \in V_{+}(I)_{k}$ in $\boldsymbol{X}_{k}$. Then Corollary 3.5 has the following translation.

Proposition 3.13. With the notation of Corollary 3.5, after a possible finite extension of $K$, there exists $\mathfrak{X} \in \boldsymbol{X}^{\mathrm{ss}}(\mathcal{O})$ such that $\mathfrak{X}_{K} \in G(K) \cdot \mathrm{x}$ and $\underline{I}(\mathfrak{X})$ is a minimal representative of $\underline{I}(\mathrm{x})$.

Proof. After possibly a finite extension of $K$, let $\mathfrak{x} \in \boldsymbol{X}^{\mathrm{ss}}(\mathcal{O})$ be as given by Corollary 3.5. As $V_{+}(I)=\emptyset$ in Proj $B^{G}$, we have $\boldsymbol{X}^{\mathrm{ss}}=\cup_{f \in I} D_{+}(f)$, hence $\mathfrak{X} \in D_{+}(f)$ for some $f \in I$. Therefore $v(f(\mathfrak{x})) \in \mathcal{O}^{*}$ and $\underline{I}(\mathfrak{X})$ is a minimal representative of $\underline{I}(\mathrm{x})$.

Let $d>2, n \geq 1$ be integers and $N=\left(\begin{array}{c}n+d \\ d\end{array}\right)$. Let $\boldsymbol{X} \simeq \mathbb{P}^{N}=\operatorname{Proj} \mathbb{Z}[\underline{x}]$, the space of hypersurfaces of degree $d$ in $\mathbb{P}^{n}$ under the classical action of $G=\mathrm{SL}_{n+1, \mathbb{Z}}$. As $\mathbb{Z}$ is a $\mathrm{PID}$, any $X \in \boldsymbol{X}(\mathbb{Z})$ is represented by an $(n+1)$-ary form $F$, unique up to multiplication by \pm 1 .

Note that $\mathbb{Z}$ is excellent and pictorsion and that the scheme $\boldsymbol{X}$ satisfies the hypotheses of Corollary 3.12 hence $\mathbb{Z}[\underline{x}]^{G}$ admits a HSOP over $\mathbb{Z}$. Moreover, there exists an invariant $D_{\ell} \in \mathbb{Z}[\underline{x}]^{G}$ of degree $\ell=$ $(n+1)(d-1)^{n}$, called the discriminant, such that any hypersurface $F=0$ over a ring $R$ is smooth if and only if $D_{\ell}(F)$ is invertible in $R$ [GKZ94, p.426], [Dem12]. Over any discrete valuation ring $\mathcal{O}, \mathcal{O}[\underline{x}]^{G_{\mathcal{O}}}$ is finitely generated as $\mathcal{O}$-algebra by Remark 3.6, and any HSOP of $\mathbb{Z}[\underline{x}]^{G}$ then gives rise to a HSOP of $\mathcal{O}[\underline{x}]^{G_{\mathcal{O}}}$ according to remark 3.10 .

Corollary 3.14. Let $K$ be a discrete valuation field with valuation $v$, valuation ring $\mathcal{O}$ and residue field $k$ of characteristic $p \geq 0$. Let $\underline{I}$ be a HSORG for the graded algebra $\mathbb{Z}[\underline{x}]^{\mathrm{SL}_{n+1, \mathbb{Z}}}$ for hypersurfaces of degree $d$ in $\mathbb{P}^{n}$. Let $X / K: F=0$ be a smooth hypersurface of degree $d$ in $\mathbb{P}^{n}$ and $D_{\ell}$ the discriminant as defined above. Then $v_{I}\left(D_{\ell}(F)\right)=0$ if and only if, after a possible finite extension of $K$, there exists a hypersurface $\mathcal{X} / \mathcal{O}$ such that $\mathcal{X}_{K} \simeq X$ and $\mathcal{X}_{k}$ is a smooth hypersurface of degree $d$.

Proof. Let $X / K: F=0$ be a smooth hypersurface of $\mathbb{P}^{N}$ of degree $d$. By [MF82, Prop.4.2], $X / K$ is GIT-stable. After a possible finite extension of $K$, by Proposition 3.13, there exists a hypersurface $\mathcal{X} / \mathcal{O}: F_{0}=0$ in $\mathbb{P}^{N}$ of degree $d$, isomorphic to $X$ over $K$ and such that $\underline{I}\left(F_{0}\right)$ is a minimal representative over $\mathcal{O}$. Therefore if $v_{I}\left(D_{\ell}(F)\right)=0$, then $v\left(D_{\ell}\left(F_{0}\right)\right)=0$ and $\mathcal{X}_{k}$ is a smooth hypersurface of degree $d$. This proves the direct implication. For the converse implication, if $\mathcal{X} / \mathcal{O}: F_{0}=0$ is smooth, necessarily in $\mathbb{P}^{n}$ of degree $d$, then $D_{\ell}\left(\bar{F}_{0}\right) \neq 0$ i.e. $v\left(D_{\ell}\left(F_{0}\right)\right)=0$ which implies, since $\mathcal{X}_{K} \simeq X$, that $v_{I}\left(D_{\ell}(F)\right)=0$ as well.

As a particular case, we get our Theorem 1.9. Indeed, notice that by the discussions in $\S 2.1$, if a smooth quartic curve $X / K$ has potentially good quartic reduction, then after possibly a finite extension of $K, X$ is isomorphic to the generic fiber of some smooth quartic curve over $\mathcal{O}$.

Theorem 3.15 (See Theorem 1.9). Let $K$ be a discrete valuation field with valuation $v$, valuation ring $\mathcal{O}$ and residue field $k$ of characteristic $p \geq 0$. Let $\underline{I}$ be a list of invariants which is a HSORG for the ternary quartic forms under the action of $\mathrm{SL}_{3, \mathcal{O}}$. Then a smooth plane quartic $C / K: F=0$ has potentially good quartic reduction if and only if $v_{I}\left(D_{27}(F)\right)=0$.

Remark 3.16. It is unclear how to effectively compute the potential smooth quartic model. In some examples, the extension of $K$ to be considered can be huge. For instance, $y^{3}=x^{4}-x$ has good quartic reduction over an extension of minimal degree 108 of $\mathbb{Q}_{3}$ (Bouw-Wewers, private communication). 
The previous result will be effective once we have determine a HSOP. This is the main result of Section 4.1. Meanwhile, let us work out a simpler case, which will also be useful for the characterization of potentially good hyperelliptic reduction. When $p \neq 2,3,5$ and 7, [LR12, Prop.1.9] shows that the set of Shioda invariants $j_{2}, \ldots, j_{10}$ is a HSORG over $\mathcal{O}$ for the graded algebra of invariants of binary octics under the action of $\mathrm{SL}_{2, \mathcal{O}}$. Actually, taking into account that the vanishing of $j_{2}, \ldots, j_{7}$ implies the vanishing of the discriminant $D_{14}$, the proof of loc. cit. shows that $\underline{\mathrm{Sh}}=\left(j_{2}, \ldots, j_{7}\right)$ is a HSOP over $\mathcal{O}$ when $p=0$ or $p>7$.

Corollary 3.14 specializes in the following.

Corollary 3.17. Let $K$ be a discrete valuation field with valuation $v$, valuation ring $\mathcal{O}$ and residue field $k$ of characteristic $p \neq 2,3,5$ and 7 . Let $C: y^{2}=f(x)$ over $K$ be a hyperelliptic curve of genus 3 with $f \in \mathcal{O}[x]$. Then $C$ has potentially good reduction if and only if $v_{\mathrm{Sh}}\left(D_{14}(f)\right)=0$, with $\underline{\mathrm{Sh}}=\left(j_{2}, \ldots, j_{7}\right)$.

Remark 3.18. For binary octics, Basson [Bas15] exhibits a HSOP of degree $(3,4,5,6,10,14)$ when $p=7$ and one of degree $(4,5,6,7,8,9)$ when $p=3$. Similar techniques for $p=5$ lead to a HSOP over $\mathcal{O}$ of degree $(4,6,6,12,14,20)$. We can therefore extend Corollary 3.17 to all characteristics different from 2 using these sets instead.

\section{Homogeneous System of PARAmeters over $\mathcal{O}$}

4.1. Computation of the various HSOP. Let $\mathcal{O}$ be an DVR with residue field $k$ of characteristic $p$. In order to make Theorem 1.9 effective, we need an explicit HSOP over $\mathcal{O}$ for ternary quartic forms under the action of $\mathrm{SL}_{3, \mathcal{O}}$. We have seen in Section 3.3 that such a HSOP exists and is even defined over $\mathbb{Z}$. Dixmier proves [Dix87] that the 7 invariants $I_{3}, I_{6}, I_{9}, I_{12}, I_{15}, I_{18}$ and $I_{27}$ form a HSOP over a field of characteristic 0 . The result does not carry in characteristic $p>0$ for all $p$ but the strategy he followed is valid in positive characteristic, although it requires to be handled with care.

We work out the computations with the computer algebra software MAGMA. The invariants are given by polynomials over $\mathbb{Q}$ in the Dixmier-Ohno invariants. They are found by an evaluation/interpolation strategy over $\mathbb{Z} / p^{n} \mathbb{Z}$ for increasing powers $n$ (the largest computation being in degree 81 over $3^{19}$ ). We will not give details about this search here. Once given, it is indeed "enough" to check that that the invariants we obtained satisfy two conditions: 1) as polynomials in the 15 coefficients of a ternary quartic forms, they have integral coefficients; 2) once reduced modulo $p$, these polynomials form a HSOP over $\overline{\mathbb{F}}_{p}$. We could prove these two facts for our invariants in all characteristics $p \neq 3$. Let us say quickly some words about 1). For a given characteristic, one can transform a general plane quartic into a quartic with only 7 parameters by an integral change of variables (when $p>3$, one can take Shioda's models in [Shi93]). When the degree of the invariant is less or equal to 27, it is then possible to write down the invariant extensively in these 7 variables and check directly the integrality of each coefficient. For $p=3$, we cannot prove integrality for the degree 54 and 81 invariants and we therefore only present the result as Conjecture 4.2. Dealing with 2) is somehow classical as we will see in the proof of the following result.

Theorem 4.1. Let $\mathcal{O}$ be a DVR with residue field of characteristic $p \neq 3$. A HSOP over $\mathcal{O}$ for ternary quartic forms under the action of $\mathrm{SL}_{3, \mathcal{O}}$ is formed by

- $\left(I_{3}^{(2)}, I_{9}^{(2)}, I_{12}^{(2)}, I_{15}^{(2)}, I_{18}^{(2)}, I_{24}^{(2)}, D_{27}\right)$ when $p=2$. The large expressions can be found in [LLLR19, DixmierHSOP.txt]; 
- $\left(I_{3}, I_{6}, I_{9}^{(5)}, I_{12}, J_{15}^{(5)}, I_{18}, I_{27}\right)$ where $I_{9}^{(5)}=\left(J_{9}+3 I_{9}\right) / 5$ and

$$
\begin{aligned}
J_{15}^{(5)}=\left(-64 I_{3}{ }^{3} I_{6}-24 I_{3} I_{6}{ }^{2}+39 I_{3} I_{12}-11 I_{3} J_{12}+42 I_{6} I_{9}-21 I_{6} J_{9}-1143 I_{15}+J_{15}\right) / 5^{3} & \\
& +\left(253 I_{3}{ }^{2} I_{9}-79 I_{3}{ }^{2} J_{9}\right) / 5^{4} \text { when } p=5 ;
\end{aligned}
$$

- $\left(I_{3}, I_{6}, I_{9}-J_{9}, I_{12}, I_{15}, I_{18}, I_{27}\right)$ when $p=7,19,47,277$ and 523;

- $\left(I_{3}, I_{6}, I_{9}, I_{12}, I_{15}, I_{18}, I_{27}\right)$ otherwise.

In particular for $p \geq 7$, the Dixmier-Ohno invariants form a HSORG.

Proof. By Remark 3.10, it is enough to prove that the previous sets are HSOP over the algebraic closure of the residue field of $\mathcal{O}$. To do this, we first find all ternary quartic forms $F$ in characteristic $p$ such that $I(F)=0$ for all $I \in \underline{I}$, where $\underline{I}$ is the set of invariants given in the theorem. We write $F$ as

$$
\begin{array}{r}
a_{04} x_{2}^{4}+a_{13} x_{1} x_{2}{ }^{3}+a_{22} x_{1}{ }^{2} x_{2}{ }^{2}+a_{31} x_{1}{ }^{3} x_{2}+a_{40} x_{1}{ }^{4}+\left(a_{03} x_{2}{ }^{3}+a_{12} x_{1} x_{2}{ }^{2}+a_{21} x_{1}{ }^{2} x_{2}+a_{30} x_{1}{ }^{3}\right) x_{3} \\
+\left(a_{02} x_{2}{ }^{2}+a_{11} x_{1} x_{2}+a_{20} x_{1}{ }^{2}\right) x_{3}{ }^{2}+\left(a_{01} x_{2}+a_{10} x_{1}\right) x_{3}{ }^{3}+a_{00} x_{3}{ }^{4} .
\end{array}
$$

Then, the set $\underline{I}$ is a HSOP in characteristic $p$ if and only if any such $F$ is GIT-unstable, or equivalently $F$ has a triple point or consists of a cubic and an inflectional tangent line [Mum77, §1.12]. Let us start with $p \neq 2$ (and 3). Since $I_{27}(F)=2^{40} D_{27}(F)=0$, the quartic $F=0$ has a singular point, that we can, up to a linear change of variables, set to $\Pi=(0: 0: 1)$. Following step by step [Dix87], we can use the

\begin{tabular}{|c|c|c|c|}
\hline Case & \multicolumn{2}{|l|}{ Condition } & $F\left(x_{1}, x_{2}, x_{3}\right)$ \\
\hline 0 & \multicolumn{2}{|l|}{$\Pi$ of order $\geqslant 3$} & $\begin{array}{c}a_{04} x_{2}{ }^{4}+a_{13} x_{1} x_{2}{ }^{3}+a_{22} x_{1}{ }^{2} x_{2}{ }^{2}+a_{31} x_{1}{ }^{3} x_{2}+a_{40} x_{1}{ }^{4}+ \\
\left(a_{03} x_{2}{ }^{3}+a_{12} x_{1} x_{2}{ }^{2}+a_{21} x_{1}{ }^{2} x_{2}+a_{30} x_{1}{ }^{3}\right) x_{3} \\
\end{array}$ \\
\hline 1.1 & \multirow{2}{*}{$\begin{array}{l}\Pi \text { of order } 2 \& \\
x_{1}=0 \text { is the only } \\
\text { tangent at } \Pi\end{array}$} & $a_{03}=0$ & $\begin{array}{c}a_{04} x_{2}^{4}+a_{13} x_{2}^{3} x_{1}+a_{22} x_{2}^{2} x_{1}^{2}+a_{31} x_{2} x_{1}^{3}+a_{40} x_{1}^{4}+ \\
\left(a_{12} x_{2}{ }^{2} x_{1}+a_{21} x_{2} x_{1}{ }^{2}+a_{30} x_{1}^{3}\right) x_{3}+x_{1}{ }^{2} x_{3}{ }^{2}\end{array}$ \\
\hline 1.2 & & $a_{03} \neq 0$ & $\begin{array}{c}a_{04} x_{2}{ }^{4}+a_{13} x_{2}{ }^{3} x_{1}+a_{22} x_{2}{ }^{2} x_{1}{ }^{2}+a_{31} x_{2} x_{1}{ }^{3}+a_{40} x_{1}{ }^{4}+ \\
x_{2}{ }^{3} x_{3}+x_{1}{ }^{2} x_{3}{ }^{2}\end{array}$ \\
\hline 2.1 & \multirow{3}{*}{$\begin{array}{l}\Pi \text { of order } 2 \& \\
x_{1}=0, x_{2}=0 \text { are } \\
\text { both tangent at } \Pi\end{array}$} & $\begin{array}{c}a_{03} \neq 0 \\
\& a_{30} \neq 0\end{array}$ & $\begin{array}{c}a_{04} x_{2}{ }^{4}+a_{13} x_{2}{ }^{3} x_{1}+a_{22} x_{2}{ }^{2} x_{1}{ }^{2}+a_{31} x_{2} x_{1}{ }^{3}+a_{40} x_{1}{ }^{4}+ \\
\left(x_{2}{ }^{3}+x_{1}{ }^{3}\right) x_{3}+x_{2} x_{1} x_{3}{ }^{2}\end{array}$ \\
\hline 2.2 & & $\begin{array}{c}a_{03}=0 \\
\& a_{30} \neq 0\end{array}$ & $\begin{array}{c}a_{04} x_{2}^{4}+a_{13} x_{2}{ }^{3} x_{1}+a_{22} x_{2}{ }^{2} x_{1}{ }^{2}+a_{31} x_{2} x_{1}{ }^{3}+a_{40} x_{1}{ }^{4}+ \\
x_{1}{ }^{3} x_{3}+x_{2} x_{1} x_{3}{ }^{2}\end{array}$ \\
\hline 2.2 & & $\begin{array}{c}a_{03}=0 \\
\& a_{30}=0\end{array}$ & $\begin{array}{c}a_{04} x_{2}^{4}+a_{13} x_{2}^{3} x_{1}+a_{22} x_{2}^{2} x_{1}^{2}+a_{31} x_{2} x_{1}^{3}+a_{40} x_{1}^{4}+ \\
x_{2} x_{1} x_{3}^{2}\end{array}$ \\
\hline
\end{tabular}
remaining degrees of freedom to restrict to the six different cases that we sum up in Table 1 and that we will consider one after the other. We checked that the linear transformations behind these normalizations are valid over fields of characteristic $p \geqslant 5$ and 0 (see [LLLR19, HSOPDetailedProof.txt]).

TABLE 1. Singular quartics in characteristic $p \geqslant 5$ and 0

- Case 0 yields quartics with $\Pi$ being a triple point and these curves are GIT-unstable.

- Case 1.1 is also easily manageable. We use the computer algebra software MaGma to shortcut some cumbersome computations (that Dixmier made by hand over the rationals!). In particular, we compute a Gröbner basis over $\mathbb{Z}$ (based on the computation of normal Hermite forms of integral Macaulay matrices) of the algebraic system $\{I(F)=0\}_{I \in \underline{I}}$ for the following lexicographic order:

$$
a_{30}>a_{22}>a_{21}>a_{31}>a_{13}>a_{40}>a_{04} .
$$


We get $\left\{a_{12}{ }^{8}+144 a_{04}{ }^{4}, a_{12}{ }^{4} a_{04}+144 a_{04}{ }^{3}, 2 a_{12}{ }^{2}+12 a_{04}, 360 a_{04}{ }^{3}, 720 a_{04}{ }^{2}\right\}$. In characteristic $p>5$ and $p=0$, we thus have $a_{04}=a_{12}=0$. This yields quartics $F=0$ which are the union of a cubic curve and an inflectional tangent at $\Pi$, which we can assume to be $x_{1}=0$, thus GIT-unstable quartics. The exceptional prime $p=5$ will be handled later on its own.

- Case 1.2 is done in a similar manner. Gröbner basis can also be computed over $\mathbb{Z}$. When $p>5$ and $p=0$, we derive from it that $a_{04}=a_{31}=a_{22}=0$, and $\left\{a_{13}{ }^{2}+9953670985 a_{40}, 2^{4} \cdot 3^{13} \cdot 5^{2} \cdot 7 \cdot 19 \cdot 47 a_{40}\right\}$. Thus, for $p \neq 2,3,5,7,19$ and 47, we have $a_{13}=a_{40}=0$. This yields quartics of the same type as in Case 1.1, and thus, GIT-unstable. These exceptional primes will be handled later on their own.

- Case 2.1 is the most difficult case, at least computationally speaking. From $I_{3}(F)=I_{6}(F)=0$, we easily deduce that $a_{22}=-9 / 2$ and $a_{04} a_{40}=9 / 4$. Now, the equations $I(F)=0$ for the other invariants $I$ yield more algebraic relations between the remaining variables, but of higher degrees, and we were not able anymore to compute an integral Gröbner basis. Instead, we find sufficient conditions on the remaining $a_{i j}$ by solving with resultants. We eliminate, first $a_{04}$, then $a_{40}$ and finally $a_{31}$ (resp. $a_{13}, a_{31}$ and $a_{04}$ ), and obtain a polynomial of degree 48 in $a_{13}$ (resp. of degree 28 in $a_{40}$ ),

$$
N_{13}\left(4 a_{13}+15\right)^{16}\left(16 a_{13}^{2}-60 a_{13}+225\right)^{16} \quad\left(\text { resp. } N_{40} a_{40}^{4}\left(2 a_{40}+3\right)^{8}\left(4 a_{40}^{2}-6 a_{40}+9\right)^{8}\right),
$$

where $N_{13}$ (resp. $N_{40}$ ) is an integer, too large to be completely factorized. We can have $N_{13}$ and $N_{40}$ both equal to zero modulo $p$, but this can only happen for the few prime divisors $p$ of $\operatorname{gcd}\left(N_{13}, N_{40}\right)$. These cases can be easily studied independently (see the end of the proof). Let us assume from now that it is not the case, i.e. $p \neq 2,3,5,7,13,43,47,89,277$ and 523 . Either $N_{13} \neq 0$ or $N_{40} \neq 0$.

- If $N_{13} \not \equiv 0 \bmod p$, then we have two possibilities :

$-4 a_{13}+15=0$, and with a couple of additional resultants, we show that $2 a_{40}+3=0$ when $p \neq 2,3,13,1613,3469$ and 6 other 5-24 digit primes;

$-16 a_{13}^{2}-60 a_{13}+225=0$, and this implies that $4 a_{40}^{2}-6 a_{40}+9=0$ when additionally $p \neq 37,61$ and 210037 .

- If $N_{40} \not \equiv 0 \bmod p$, then we have the two symmetric possibilities :

$-2 a_{40}+3=0$, which implies that $4 a_{13}+15=0$ when additionally $p \neq 53,4969$ and 3 new 7-19 digit primes;

$-4 a_{40}^{2}-6 a_{40}+9=0$, which implies $16 a_{13}^{2}-60 a_{13}+225=0$ when $p$ is not one of the primes above.

In other words, except modulo the few primes $p$ that we have listed above, we have either

$$
\begin{aligned}
& a_{31}=-15 / 4, a_{13}=-15 / 4, a_{40}=-3 / 2 \text { and } a_{04}=-3 / 2 \text { or } \\
& \qquad a_{04}^{2}-3 / 2 a_{04}+9 / 4=0, a_{40}=-a_{04}+3 / 2, a_{13}=-5 / 2 a_{04}+15 / 4 \text { and } a_{31}=5 / 2 a_{04} .
\end{aligned}
$$

In both cases, this yields quartics $F=0$ which are the union of a cubic curve and an inflectional tangent, thus GIT-unstable.

- Cases 2.2 and 2.3 are much easier and can be handled again with integral Gröbner basis computations. They do not yield additional exceptional primes. We omit the details.

To end the proof, we have to consider each exceptional prime $p>3$ one by one, and check over $\overline{\mathbb{F}}_{p}$ the GIT-stability of the quartics $F$ that cancel the given set of invariants. Since we have to deal now with Gröbner basis computations in finite fields, and no longer over $\mathbb{Z}$, these computations can be easily done with Magma. 
For $p=2$, a similar work can be done to obtain the same six cases of Table 1, with slightly distinct forms $F$ (see Table 2 and [LLLR19, File HSOPDetailedProof.txt]). Then, a direct Gröbner basis computations easily yields the subset of these models that cancel our set of 7 invariants. The associated curves either have a singular point of order 3, or are the union of a cubic curve and an inflectional tangent. They thus all are GIT-unstable and the set is a HSOP.

\begin{tabular}{|c|c|c|c|}
\hline Case & \multicolumn{2}{|l|}{ Condition } & $F\left(x_{1}, x_{2}, x_{3}\right)$ \\
\hline 0 & \multicolumn{2}{|l|}{$\Pi$ of order $\geqslant 3$} & $\begin{array}{c}a_{04} x_{2}{ }^{4}+a_{13} x_{1} x_{2}{ }^{3}+a_{22} x_{1}{ }^{2} x_{2}{ }^{2}+a_{31} x_{1}{ }^{3} x_{2}+a_{40} x_{1}{ }^{4}+ \\
\left(a_{03} x_{2}{ }^{3}+a_{12} x_{1} x_{2}{ }^{2}+a_{21} x_{1}{ }^{2} x_{2}+a_{30} x_{1}^{3}\right) x_{3}\end{array}$ \\
\hline 1.1 & \multirow{2}{*}{$\begin{array}{l}\Pi \text { of order } 2 \& \\
x_{1}=0 \text { is the only } \\
\text { tangent at } \Pi\end{array}$} & $a_{03}=0$ & $\begin{array}{r}x_{1}\left(a_{13} x_{2}^{3}+a_{22} x_{1} x_{2}^{2}+a_{31} x_{1}^{2} x_{2}+a_{40} x_{1}^{3}+\right. \\
\left.\left(a_{21} x_{1} x_{2}+a_{30} x_{1}^{2}\right) x_{3}+x_{1} x_{3}^{2}\right)\end{array}$ \\
\hline 1.2 & & $a_{03} \neq 0$ & $x_{2}\left(a_{31} x_{1}^{3}+x_{2}^{2} x_{3}+x_{1} x_{3}^{2}\right)$ \\
\hline 2.1 & \multirow{3}{*}{$\begin{array}{l}\Pi \text { of order } 2 \& \\
x_{1}=0, x_{2}=0 \text { are } \\
\text { both tangent at } \Pi\end{array}$} & $\begin{array}{c}a_{03} \neq 0 \\
\& a_{30} \neq 0\end{array}$ & $\begin{array}{c}x_{3}\left(x_{2}^{3}+a_{12} x_{1} x_{2}^{2}+\left(a_{12}+1\right) x_{1}^{2} x_{2}+x_{1}^{3}+x_{1} x_{2} x_{3}\right) \\
\text { or } \quad x_{3}\left(x_{2}^{3}+x_{1} x_{2}^{2}+x_{1}^{2} x_{2}+x_{1}^{3}+x_{1} x_{2} x_{3}\right) \\
\end{array}$ \\
\hline 2.2 & & $\begin{array}{c}a_{03}=0 \\
\& a_{30} \neq 0\end{array}$ & $\begin{aligned} x_{1}\left(a_{13} x_{2}^{3}+a_{22} x_{1}\right. & x_{2}^{2}+a_{31} x_{1}^{2} x_{2}+a_{40} x_{1}^{3} \\
& \left.+\left(a_{21} x_{1} x_{2}+x_{1}^{2}\right) x_{3}+x_{2} x_{3}^{2}\right)\end{aligned}$ \\
\hline 2.2 & & $\begin{array}{c}a_{03}=0 \\
\& a_{30}=0\end{array}$ & $\begin{array}{r}x_{1}\left(a_{13} x_{2}^{3}+a_{22} x_{1} x_{2}^{2}+a_{31} x_{1}^{2} x_{2}+a_{40} x_{1}^{3}\right. \\
\left.+a_{21} x_{1} x_{2} x_{3}+x_{2} x_{3}^{2}\right)\end{array}$ \\
\hline
\end{tabular}

TABLE 2. Singular quartics in characteristic $p=2$

We encountered more difficulties to find a HSOP in characteristic 3. Especially, it is only after a lengthy computation that we finally manage to find an invariant, of degree 81, which does not vanish on the orbit of the GIT-semi-stable quartic $-x_{2}^{3} x_{3}+x_{1}^{4}+x_{1}^{2} x_{3}^{2}$. This finally yields a HSOP formed of explicit invariants $I_{3}^{(3)}, I_{27}^{(3)}=I_{27}, J_{27}^{(3)}, I_{36}^{(3)}, J_{36}^{(3)}, I_{54}^{(3)}$ and $I_{81}^{(3)}$ (we also refer to [LLLR19, DixmierHSOP.txt] for their polynomial expressions in the Dixmier-Ohno invariants).

We do not have a proof that the expressions of $I_{54}^{(3)}$ and $I_{81}^{(3)}$ in terms of the 15 coefficients are polynomials with integer coefficients. Because of the degree of these expressions, it is simply impossible to even write this expression which has about $2^{56}$ monomials. We hope that this integrality property can be proved in a near future in a different way. Meanwhile, supported by extensive experiments that we made on random and one parameter families of quartics over $\mathbb{Z}$, we state the following conjecture.

Conjecture 4.2 (Integrality hypothesis). The invariants $I_{54}^{(3)}$ and $I_{81}^{(3)}$ have integral coefficients.

Theorem 4.3. Let $\mathcal{O}$ be a DVR with residue field of characteristic $p=3$. Under Conjecture 4.2, the invariants $I_{3}^{(3)}, I_{27}^{(3)}=I_{27}, J_{27}^{(3)}, I_{36}^{(3)}, J_{36}^{(3)}, I_{54}^{(3)}$ and $I_{81}^{(3)}$ form a HSOP over $\mathcal{O}$ for ternary quartic forms under the action of $\mathrm{SL}_{3, \mathcal{O}}$.

Proof. The strategy follows the same lines as for $p=2$. One shall this time replace Table 1 by Table 3 (see [LLLR19, File HSOPDetailedProof.txt] for a detailed proof of these cases).

Remark 4.4. With the same assumptions as Theorem 3.15, the invariants of the special fiber $\mathcal{C}_{k}$ are the reduction modulo $\pi$ of a minimal representative of $\underline{I}(F)$. Hence, if an algorithm to reconstruct from the invariants is available, one can get an equation for $\mathcal{C}_{k}$. This is in particular the case for generic plane quartics in characteristic $p>7$ when $\underline{\mathrm{DO}} \subset \underline{I}$ using [LRS18]. 


\begin{tabular}{|c|c|c|c|}
\hline Case & \multicolumn{2}{|l|}{ Condition } & $F\left(x_{1}, x_{2}, x_{3}\right)$ \\
\hline 0 & \multicolumn{2}{|l|}{$\Pi$ of order $\geqslant 3$} & $\begin{array}{c}a_{04} x_{2}{ }^{4}+a_{13} x_{1} x_{2}{ }^{3}+a_{22} x_{1}{ }^{2} x_{2}{ }^{2}+a_{31} x_{1}{ }^{3} x_{2}+a_{40} x_{1}{ }^{4}+ \\
\left(a_{03} x_{2}{ }^{3}+a_{12} x_{1} x_{2}{ }^{2}+a_{21} x_{1}{ }^{2} x_{2}+a_{30} x_{1}{ }^{3}\right) x_{3}\end{array}$ \\
\hline 1.1 & \multirow{2}{*}{$\begin{array}{l}\Pi \text { of order } 2 \& \\
x_{1}=0 \text { is the only } \\
\text { tangent at } \Pi\end{array}$} & $a_{03}=0$ & $\begin{aligned} x_{1}\left(a_{13} x_{2}^{3}+a_{22} x_{1} x_{2}^{2}+a_{31} x_{1}^{2} x_{2}+a_{40} x_{1}^{3}+\right. \\
\left.\left(a_{21} x_{1} x_{2}+a_{30} x_{1}^{2}\right) x_{3}+x_{1} x_{3}^{2}\right)\end{aligned}$ \\
\hline 1.2 & & $a_{03} \neq 0$ & $x_{3}\left(x_{2}^{3}+a_{30} x_{1}^{3}+x_{1}^{2} x_{3}\right)$ \\
\hline 2.1 & \multirow{3}{*}{$\begin{array}{l}\Pi \text { of order } 2 \& \\
x_{1}=0, x_{2}=0 \text { are } \\
\text { both tangent at } \Pi\end{array}$} & $\begin{array}{l}a_{03} \neq 0 \\
\& a_{30} \neq 0\end{array}$ & $x_{3}\left(x_{2}^{3}+x_{1}^{3}+x_{1} x_{2} x_{3}\right)$ \\
\hline 2.2 & & $\begin{array}{c}a_{03}=0 \\
\& a_{30} \neq 0\end{array}$ & $x_{1}\left(a_{31} x_{1}^{2} x_{2}+a_{40} x_{1}^{3}+x_{1}^{2} x_{3}+x_{2} x_{3}^{2}\right)$ \\
\hline 2.2 & & $\begin{array}{c}a_{03}=0 \\
\& a_{30}=0\end{array}$ & $\begin{array}{l}\quad x_{1}\left(a_{31} x_{1}^{2} x_{2}+a_{40} x_{1}^{3}+x_{2} x_{3}^{2}\right) \\
\text { or } \quad x_{2}\left(a_{04} x_{2}^{3}+a_{13} x_{1} x_{2}^{2}+x_{1} x_{3}^{2}\right)\end{array}$ \\
\hline
\end{tabular}

TABLE 3. Singular quartics in characteristic $p=3$

Example 4.5. Let us consider the quartic $X_{1}: F_{1}=0$ with

$$
\begin{gathered}
F_{1}=-4169 x_{1}^{4}-956 x_{1}^{3} x_{2}+7440 x_{1}^{3} x_{3}+55770 x_{1}^{2} x_{2}^{2}+43486 x_{1}^{2} x_{2} x_{3}+42796 x_{1}^{2} x_{3}^{2}-38748 x_{1} x_{2}^{3}-30668 x_{1} x_{2}^{2} x_{3} \\
+79352 x_{1} x_{2} x_{3}^{2}-162240 x_{1} x_{3}^{3}+6095 x_{2}^{4}+19886 x_{2}^{3} x_{3}-89869 x_{2}^{2} x_{3}^{2}-1079572 x_{2} x_{3}^{3}-6084 x_{3}^{4}
\end{gathered}
$$

from $\left[\mathrm{KLL}^{+} 18\right.$, Sec.5]. The values at $F_{1}$ of its Dixmier-Ohno invariants are

$$
\begin{aligned}
I_{3}\left(F_{1}\right) & =2^{-2} \cdot 3^{-2} \cdot 5 \cdot 13^{2} \cdot 43 \cdot 108879238253 \\
I_{6}\left(F_{1}\right) & =2^{-6} \cdot 3^{-6} \cdot 5 \cdot 13^{4} \cdot 33879904575927947128535137 \\
& \cdots \\
J_{21}\left(F_{1}\right) & =2^{-18} \cdot 3^{-16} \cdot 5 \cdot 7 \cdot 11 \cdot 13^{14} \cdot 89 \cdot 11383 \cdot \text { huge prime } \\
I_{27}\left(F_{1}\right) & =-2^{30} \cdot 5^{12} \cdot 7^{9} \cdot 13^{18} \cdot 37^{14} \cdot 15187^{14}
\end{aligned}
$$

For any prime $p$ not dividing $D_{27}\left(F_{1}\right)=2^{40} \cdot I_{27}\left(F_{1}\right), X_{1}$ has good quartic reduction. Let us now compute the valuation at $p$ of the various HSOPs we found and the normalized valuation of $D_{27}\left(F_{1}\right)$ with respect to the HSOP.

\begin{tabular}{|c|c|c|}
\hline$p$ & valuations at $p$ & normalized val. of $D_{27}\left(F_{1}\right)$ \\
\hline 2 & $2,4,6,2,11,0,70$ & $70 / 27$ \\
5 & $1,1,0,2,1,2,12$ & $12 / 27$ \\
7 & $0,0,0,0,0,0,9$ & $9 / 27$ \\
13 & $2,4,6,8,10,12,18$ & $\frac{18}{27}-\min \left(\frac{2}{3}, \frac{4}{6}, \frac{6}{9}, \frac{8}{12}, \frac{10}{15}, \frac{12}{18}, \frac{18}{27}\right)=0$ \\
37 & $0,0,0,0,0,0,14$ & $14 / 27$ \\
15187 & $0,0,0,0,0,0,14$ & $14 / 27$ \\
\hline
\end{tabular}

By using Theorem 1.9, it is easy to see that among these primes, $X_{1}$ has potentially good quartic reduction if and only if $p=13$. A minimal representative of $\underline{\mathrm{DO}}\left(F_{1}\right)$ reduces modulo 13 to $(9,2,10,5,12,6,1,9,3,3,10,8,11)$. Using [LRS18], we find that they are the invariants of the smooth plane quartic $x_{1}^{3} x_{3}+x_{1}^{2} x_{2}^{2}+x_{2}^{3} x_{3}+4 x_{3}^{4}=0$. We will resume in Example 5.4 the study of $X_{1}$ and see for which primes among $p=2,5,7,37$ and 15187 , the reduction is not potentially good. 
Remark 4.6. Finding a set of generators for the algebra of invariants in small characteristics $p$ to replace or complete the Dixmier-Ohno invariants is, as far as we know, an open problem. Notice that the situation is more subtle than for the HSOP as there may be invariants which do not come from reduction of an invariant in characteristic 0 . Over $\overline{\mathbb{F}}_{3}$, the degree- 6 homogeneous component is not of dimension 2 as in characteristic 0 , but of dimension 3 , generated by $i_{3}^{2}, i_{6}$ and $i_{6}^{\prime}$ where

$$
i_{3}=3^{3} I_{3}, \quad i_{6}=3^{2}\left(18 I_{6}-I_{3}{ }^{2}\right) \text { and } i_{6}^{\prime}=3^{5}\left(9 I_{9}-I_{3}{ }^{3}\right) / i_{3} \text {. }
$$

Notice that these expressions must be understood as the reduction of the polynomial expressions in the coefficients of a general ternary quartic form over $\mathbb{Z}$.

4.2. The case of Picard curves. As an other illustration, let us look at the well-studied family of Picard curves [BBW17, Hol95, KlcLGS20, KW05, LS16]. Since a Picard curve $C / K$ cannot be hyperelliptic (see for instance [ACGH85, p.13]), the reduction of the stable model of $C$ is either a non-hyperelliptic (Picard) curve or singular. Thus, we can get a complete characterization of the type of potential reduction with Theorem 1.9. In the present section, we go one step further and translate these conditions on the valuation of the involved invariants in human readable conditions. This study is split into several subcases. In order to shorten it, we exclude the case where the characteristic of $K$ is 3 (but the residue field can be of characteristic 3 ).

After a possible finite extension of $K$, we extend to $\mathcal{O}$ the cyclic covering to $\mathbb{P}^{1} / K$, and we can even assume that $C$ has a model $\mathcal{C}_{0}: F_{0}=0$ over $\mathcal{O}$ where

$$
\begin{aligned}
& F_{0}=-x_{2}^{3} x_{3}+x_{1}^{4}+b x_{1}^{2} x_{3}^{2}+c x_{1} x_{3}^{3}+d x_{3}^{4}, \text { when } \operatorname{Char}(K) \neq 2, \\
& F_{0}=-x_{2}^{3} x_{3}+x_{1}^{4}+a x_{1}^{3} x_{3}+b x_{1}^{2} x_{3}^{2}+c x_{1} x_{3}^{3}+d x_{3}^{4}, \text { when } \operatorname{Char}(K)=2,
\end{aligned}
$$

with $a, b, c, d \in \mathcal{O}$. Note that we could for instance let $d=0$ in the second model but the transformation to get it may require a larger extension of $K$ and we will not need this to prove our result.

To simplify the expressions, we introduce three invariants of binary quartic forms $a_{4} x^{4}+a_{3} x^{3} z+$ $a_{2} x^{2} z^{2}+a_{1} x z^{3}+a_{0} z^{4}$ under the action of $\mathrm{SL}_{2, \mathcal{O}}$, namely

$$
\begin{aligned}
& q_{2}=12 a_{0} a_{4}-3 a_{1} a_{3}+a_{2}{ }^{2}, \\
& q_{3}=72 a_{0} a_{2} a_{4}-27 a_{0} a_{3}{ }^{2}-27 a_{1}{ }^{2} a_{4}+9 a_{1} a_{2} a_{3}-2 a_{2}{ }^{3},
\end{aligned}
$$

and the discriminant $D_{6}$ which satisfies the relation $3^{3} \cdot D_{6}=4 q_{2}^{3}-q_{3}^{2}$. Let us start with the case $p \neq 2$.

Theorem 4.7. Let $K$ be a discrete valuation field with valuation $v$, valuation ring $\mathcal{O}$ and a uniformizer $\pi$. Let $k=\mathcal{O} /\langle\pi\rangle$ be the residue field of characteristic $p \neq 2$ and 3 . The curve $\mathcal{C}_{0}:-x_{2}^{3} x_{3}+G_{0}\left(x_{1}, x_{3}\right)=0$ where $G_{0}=x^{4}+b x^{2} z^{2}+c x z^{3}+d z^{4}=0$ has potentially good quartic reduction if and only if

$$
\min (6 v(b), 3 v(d)) \geq v\left(D_{6}\left(G_{0}\right)\right) .
$$

A stable model $\mathcal{C}$ is given by $\mathcal{C}:-x_{2}^{3} x_{3}+G\left(x_{1}, x_{3}\right)=0$ where

$$
G=x_{1}^{4}+b / \mathfrak{p}^{6} x_{1}^{2} x_{3}^{2}+c / \mathfrak{p}^{9} x_{1} x_{3}^{3}+d / \mathfrak{p}^{12} x_{3}^{4}=0,
$$

$\mathfrak{p}=\pi^{v\left(D_{6}\left(G_{0}\right)\right) / 36}$, and the $\operatorname{map} \mathcal{C} \rightarrow \mathcal{C}_{0}:\left(x_{1}: x_{2}: x_{3}\right) \mapsto\left(\mathfrak{p}^{3} x_{1}: \mathfrak{p}^{4} x_{2}: x_{3}\right)$.

Proof. We have

$$
v_{6}:=D_{6}\left(G_{0}\right)=2^{8} \cdot 3^{3} d^{3}-2^{7} \cdot 3^{3} b^{2} d^{2}+2^{4} \cdot 3^{3} b^{4} d+c^{2}\left(2^{4} \cdot 3^{5} b d-3^{6} c^{2}-2^{2} \cdot 3^{3} b^{3}\right)
$$


Assume the conditions (4.2), we first want to prove that $G$ is defined over $\mathcal{O}$, which boils down to $4 v(c) \geq v_{6}$. From the expression of $D_{6}\left(G_{0}\right)$ above, the conditions imply

$$
2 v(c)+v\left(2^{4} \cdot 3^{5} b d-3^{6} c^{2}-2^{2} \cdot 3^{3} b^{3}\right) \geq v_{6} .
$$

If $v(c)<v_{6} / 4$, then $v\left(2^{4} \cdot 3^{5} b d-3^{6} c^{2}-2^{2} \cdot 3^{3} b^{3}\right)<v_{6} / 2$ and we get a contradiction. Hence, the equation of $\mathcal{C}$ has integral coefficients and its discriminant

$$
D_{27}\left(-x_{2}^{3} x_{3}+G\right)=3^{9} D_{6}(G)^{2}=3^{9} D_{6}\left(G_{0}\right)^{2} / \mathfrak{p}^{72}
$$

has valuation $0 . \mathcal{C}$ is therefore a model of $\mathcal{C}_{0}$ with good quartic reduction.

Conversely, let us assume that $\mathcal{C}_{0}$ has potentially good quartic reduction. If we use the same notation for the invariants and their values at $F_{0}$ or $G_{0}$, a quick computation yields

$$
\begin{aligned}
& 0=I_{3}=I_{6}=I_{12}=J_{12}=I_{15}=J_{15}=I_{21}=J_{21}, \\
& I_{9}=2^{-12} \cdot 3^{-4}\left(8 b q_{3}+81 q_{2}^{2}\right), \\
& J_{9}=2^{-12} \cdot 3^{-4}\left(16 b q_{3}+27 q_{2}^{2}\right), \\
& I_{18}=2^{-23} \cdot 3^{-6}\left(108 b^{2} q_{2}{ }^{3}+33 b q_{3} q_{2}{ }^{2}+8 q_{2}{ }^{4}-54 D_{6} q_{2}\right), \\
& J_{18}=2^{-23} \cdot 3^{-7}\left(36 b^{2} q_{2}{ }^{3}+51 b q_{3} q_{2}{ }^{2}+16 q_{2}{ }^{4}-108 D_{6} q_{2}\right), \\
& I_{27}=2^{-40} \cdot 3^{9} D_{6}{ }^{2} .
\end{aligned}
$$

When $p \neq 5$, by the criterion of Theorem 1.9 used with the DO, we get that $v\left(I_{9}\right) \geq v\left(I_{27}\right) / 3$ and $v\left(J_{9}\right) \geq v\left(I_{27}\right) / 3$. Since $q_{2}^{2}=2^{12} \cdot 3 \cdot 5^{-1}\left(2 I_{9}-J_{9}\right)$, we get that $v\left(q_{2}\right) \geq v\left(D_{6}\right) / 3$. Furthermore since $3^{3} D_{6}=4 q_{2}^{3}-q_{3}^{2}, v\left(q_{3}\right) \geq v\left(D_{6}\right) / 2$. Specifically, at least one of these two inequalities is an equality.

If for instance $2 v\left(q_{3}\right)=v\left(D_{6}\right)$, since $b q_{3}=2^{9} \cdot 3^{4} \cdot 5^{-1}\left(3 J_{9}-I_{9}\right), v\left(3 J_{9}-I_{9}\right) / 3 \geq v\left(I_{27}\right)$ implies $3 v\left(b q_{3}\right) \geq 2 v\left(D_{6}\right)$, or equivalently $6 v(b) \geq 4 v\left(D_{6}\right)-6 v\left(q_{3}\right)=v\left(D_{6}\right)$. Moreover, since $q_{2}=b^{2}+12 d$ and $v\left(q_{2}\right) \geq v\left(D_{6}\right) / 3$, we get $v(d) \geq v\left(D_{6}\right) / 3$.

Otherwise, if $3 v\left(q_{2}\right)=v\left(D_{6}\right)$, consider the equality

$$
2^{21} \cdot 3^{4} \cdot 5^{-1} \cdot\left(2 I_{18}-3 J_{18}+\left(I_{9}-3 J_{9}\right)\left(2 I_{9}-J_{9}\right) / 20\right)=b^{2} q_{2}^{3} .
$$

We find $3 v\left(b^{2} q_{2}^{3}\right) \geq 2 v\left(I_{27}\right)$, and thus $6 v(b) \geq v\left(D_{6}\right)$ as before and we finish the proof in the same way for $d$.

When $p=5$, we use another set of invariants from Theorem 4.1, in particular $J_{9}^{(5)}=\left(J_{9}-2 I_{9}\right) / 5$. Similarly $q_{2}^{2}=-2^{12} \cdot 3 J_{9}^{(5)}$ and $b q_{3}=2^{9} \cdot 3^{4}\left(2 J_{9}^{(5)}+I_{9}^{(5)}\right)$. We can finish the proof in the same way.

We now turn to the case $p=2$ and split it into two sub-cases according to Char $(K)=2$ or not.

Theorem 4.8. Let $K$ be a discrete valuation field with valuation $v$, valuation ring $\mathcal{O}$ and a uniformizer $\pi$. Let $k=\mathcal{O} /\langle\pi\rangle$ be the residue field of characteristic $p=2$.

- Assume that char $(K)=0$. The curve $\mathcal{C}_{0}:-x_{2}^{3} x_{3}+G_{0}\left(x_{1}, x_{3}\right)=0$ where $G_{0}=x^{4}+b x^{2} z^{2}+$ $c x z^{3}+d x^{4}$ has potentially good quartic reduction if and only if

$$
6 v\left(2^{3} b\right) \geq v\left(D_{6}\left(G_{0}\right)\right) \text { and } 3 v\left(q_{2}\right) \geq v\left(D_{6}\left(G_{0}\right)\right) .
$$

- Assume that char $(K)=2$. The curve $\mathcal{C}_{0}:-x_{2}^{3} x_{3}+G_{0}\left(x_{1}, x_{3}\right)=0$ where $G_{0}=x^{4}+a x^{3} z+$ $b x^{2} z^{2}+c x z^{3}+d z^{4}$ has potentially good quartic reduction if and only if

$$
12 v(a) \geq v\left(D_{6}\left(G_{0}\right)\right) \text { and } 3 v\left(q_{2}\right) \geq v\left(D_{6}\left(G_{0}\right)\right) \text {. }
$$


Proof. We start with the case $\operatorname{char}(K)=0$. We therefore use $G_{0}=x^{4}+b x^{2} z^{2}+c x z^{3}+d z^{4}=0$ and the HSOP DO ${ }^{(2)}=\left\{I_{3}^{(2)}, I_{12}^{(2)}, \ldots, I_{27}^{(2)}\right\}$, given in Theorem 4.1 for $p=2$. If we use the same notation for the invariants and their values at $F_{0}$ or $G_{0}$, a quick computation yields

$$
\begin{aligned}
0 & =I_{3}^{(2)}=I_{12}^{(2)}=I_{15}^{(2)}=I_{24}^{(2)}, \\
I_{9}^{(2)} & =2^{3} \cdot 3^{4} b q_{3}+3^{8} q_{2}^{2}, \\
I_{18}^{(2)} & =2^{6} \cdot 3^{14} b^{2} q_{2}^{3}+2^{4} \cdot 3^{12} \cdot 11 b q_{3} q_{2}^{2}+2^{5} \cdot 3^{11} q_{3}^{2} q_{2}, \\
I_{27}^{(2)} & =D_{27}=3^{9} D_{6}^{2} .
\end{aligned}
$$

Let us first assume the conditions (4.4). Since $3^{3} \cdot D_{6}=4 q_{2}^{3}-q_{3}^{2}$, they imply that $v\left(q_{3}\right)=v\left(D_{6}\right) / 2$. Hence $v\left(I_{9}^{(2)}\right) \geq v\left(D_{6}^{2}\right) / 3, v\left(I_{18}^{(2)}\right) \geq 2 / 3 v\left(D_{6}^{2}\right)$ and therefore $v_{\mathrm{DO}^{(2)}}\left(D_{27}\right)=0$. By Theorem $1.9, \mathcal{C}_{0}$ has potentially good quartic reduction.

Now, assume that $\mathcal{C}_{0}$ has potentially good quartic reduction. As in the proof of Theorem 4.7, a trick to shortcut the computations with the valuations is to introduce more invariants than the ones in the HSOP. Here we make use of the invariant $J_{9}^{(2)}=2^{12} \cdot 3^{7} J_{9}$ which is defined over $\mathcal{O}$. From equation (4.3), it is easy to check that

$$
q_{2}^{2}=3^{-7} \cdot 5^{-1}\left(2 I_{9}^{(2)}-3 J_{9}^{(2)}\right) \text { and } 2^{3} b q_{3}=3^{-4} \cdot 5^{-1}\left(3^{2} J_{9}^{(2)}-I_{9}^{(2)}\right) .
$$

Theorem 1.9 applied to this broader set of invariants translates into

$$
v\left(q_{2}^{2}\right)=v\left(2 I_{9}^{(2)}-3 J_{9}^{(2)}\right) \geq v\left(D_{27}\right)=v\left(D_{6}^{2}\right) / 3
$$

and so we get the second condition. Similarly, $v\left(2^{3} b q_{3}\right) \geq 2 v\left(D_{6}\right) / 3$. Since $v\left(D_{6}\right)=v\left(4 q_{2}^{3}-q_{3}^{2}\right)$ implies as before $v\left(q_{3}\right)=v\left(D_{6}\right) / 2$, we get $v\left(2^{3} b\right) \geq v\left(D_{6}\right) / 6$.

It remains to consider the case $\operatorname{char}(K)=2$ with the model $G_{0}=x^{4}+a x^{3} z+b x^{2} z^{2}+c x z^{3}+d z^{4}$. We get

$$
\begin{aligned}
0 & =I_{3}^{(2)}=I_{12}^{(2)}=I_{15}^{(2)}=I_{24}^{(2)}, \\
I_{9}^{(2)} & =a^{2} q_{3}+q_{2}^{2}, \\
I_{18}^{(2)} & =a^{4} q_{2}^{3}, \\
I_{27}^{(2)} & =D_{27}=D_{6}^{2}=q_{3}^{4} .
\end{aligned}
$$

When the conditions (4.5) are satisfied, it is easy to check that $v\left(I_{9}^{(2)}\right) \geq v\left(D_{6}^{2}\right) / 3$ and $v\left(I_{18}^{(2)}\right) \geq 2 v\left(D_{6}^{2}\right) / 3$ so $\mathcal{C}_{0}$ has potentially good quartic reduction. Conversely, making use of the invariant $J_{9}^{(2)}=q_{2}^{2}$, we find $v\left(q_{2}\right) \geq v\left(D_{6}\right) / 3$ and then since $v\left(I_{9}^{(2)}\right) \geq v\left(D_{6}^{2}\right) / 3$ and $v\left(q_{3}\right)=v\left(D_{6}\right) / 2$, we get $v(a) \geq v\left(D_{6}\right) / 12$.

Finally we look at the case $p=3$ (and $\operatorname{char}(K)=0$ ).

Theorem 4.9. Let $K$ be a discrete valuation field of characteristic 0 with valuation $v$, valuation ring $\mathcal{O}$ and a uniformizer $\pi$. Let $k=\mathcal{O} /\langle\pi\rangle$ be the residue field of characteristic $p=3$. Under Conjecture 4.2, the curve $\mathcal{C}_{0}:-x_{2}^{3} x_{3}+G_{0}\left(x_{1}, x_{3}\right)=0$ where $G_{0}=x^{4}+b x^{2} z^{2}+c x z^{3}+d z^{4}$ has potentially good quartic reduction if and only if

$$
6 v\left(3^{5 / 4} b\right) \geq v\left(D_{6}\left(G_{0}\right)\right) \text { and } 2 v\left(q_{3}\right) \geq v\left(3^{5} D_{6}\left(G_{0}\right)\right) .
$$

Proof. We use the HSOP DO ${ }^{(3)}=\left\{I_{3}^{(3)}, I_{27}^{(3)}, \ldots, I_{81}^{(3)}\right\}$, given in Theorem 4.3. In the case of the model $\mathcal{C}_{0}$ we get in particular $I_{3}^{(3)}=0$ and $I_{27}^{(3)}=D_{27}=3^{9} D_{6}^{2}$. Let us define $\nu:=v\left(D_{6}\left(Q_{0}\right)\right) / 6$. 
Let us first assume the conditions (4.6), i.e. $q_{3}=O\left(3^{5 / 2} \pi^{3 \nu}\right)$. Since $3^{3} \cdot D_{6}=4 q_{2}^{3}-q_{3}^{2}$, it implies that $v\left(q_{2}\right)=v\left(3 \pi^{2 \nu}\right)$ and $D_{6}=4\left(q_{2} / 3\right)^{3}+O\left(3^{2} \pi^{6 \nu}\right)$. Hence, a quick computation shows that

$$
\begin{aligned}
J_{27}^{(3)}= & O\left(3^{14.5} \pi^{9 \nu}\right) \cdot b^{3}+O\left(3^{13} \pi^{10 \nu}\right) \cdot b^{2}+O\left(3^{11.5} \pi^{11 \nu}\right) \cdot b+O\left(3^{9} \pi^{12 \nu}\right), \\
I_{36}^{(3)}= & O\left(3^{17} \pi^{12 \nu}\right) \cdot b^{4}+O\left(3^{17.5} \pi^{13 \nu}\right) \cdot b^{3}+O\left(3^{16} \pi^{14 \nu}\right) \cdot b^{2}+O\left(3^{14.5} \pi^{15 \nu}\right) \cdot b+O\left(3^{13} \pi^{16 \nu}\right), \\
J_{36}^{(3)}= & O\left(3^{17} \pi^{12 \nu}\right) \cdot b^{4}+O\left(3^{17.5} \pi^{13 \nu}\right) \cdot b^{3}+O\left(3^{16} \pi^{14 \nu}\right) \cdot b^{2}+O\left(3^{14.5} \pi^{15 \nu}\right) \cdot b+O\left(3^{13} \pi^{16 \nu}\right), \\
I_{45}^{(3)}= & O\left(3^{22.5} \pi^{15 \nu}\right) \cdot b^{5}+O\left(3^{21} \pi^{16 \nu}\right) \cdot b^{4}+O\left(3^{20.5} \pi^{17 \nu}\right) \cdot b^{3}+O\left(3^{20} \pi^{18 \nu}\right) \cdot b^{2}+O\left(3^{17.5} \pi^{19 \nu}\right) \cdot b+O\left(3^{16} \pi^{20 \nu}\right), \\
I_{81}^{(3)}= & O\left(3^{40.5} \pi^{27 \nu}\right) \cdot b^{9}+O\left(3^{38} \pi^{28 \nu}\right) \cdot b^{8}+O\left(3^{37.5} \pi^{29 \nu}\right) \cdot b^{7}+O\left(3^{36} \pi^{30 \nu}\right) \cdot b^{6}+O\left(3^{34.5} \pi^{31 \nu}\right) \cdot b^{5} \\
& +O\left(3^{34} \pi^{32 \nu}\right) \cdot b^{4}+O\left(3^{32.5} \pi^{33 \nu}\right) \cdot b^{3}+O\left(3^{31} \pi^{34 \nu}\right) \cdot b^{2}+O\left(3^{29.5} \pi^{35 \nu}\right) \cdot b+O\left(3^{29} \pi^{36 \nu}\right) .
\end{aligned}
$$

Since $b=O\left(3^{-1.25} \pi^{v}\right)$, we obtain $J_{27}^{(3)}=O\left(3^{9} \pi^{12 \nu}\right), I_{36}^{(3)}=O\left(3^{13} \pi^{16 \nu}\right), J_{36}^{(3)}=O\left(3^{12} \pi^{16 \nu}\right), I_{45}^{(3)}=$ $O\left(3^{16} \pi^{20 \nu}\right)$ and $I_{81}^{(3)}=O\left(3^{28} \pi^{36 \nu}\right)$. By Theorem $1.9, \mathcal{C}_{0}$ has potentially good quartic reduction.

Conversely, let us assume that $\mathcal{C}_{0}$ has potentially good quartic reduction. So $I_{81}^{(3)}=O\left(3^{27} \pi^{36 \nu}\right)$. Since, $I_{81}^{(3)} \equiv q_{2} \bmod 3^{4}$, we have $q_{2}=O(3)$. Furthermore $I_{81}^{(3)} \equiv 3^{18}\left(D_{6}-4\left(q_{2} / 3\right)^{3}\right)^{6} \bmod 3^{20}$. We thus must have $D_{6}=4\left(q_{2} / 3\right)^{3}+O\left(3^{2}\right)$. Since $3^{3} \cdot D_{6}=4 q_{2}^{3}-q_{3}^{2}$, it further implies $q_{2}=O\left(3 \pi^{2 \nu}\right)$ and $q_{3}=O\left(3^{5 / 2} \pi^{3 \nu}\right)$. By hypothesis, we also must have $J_{27}^{(3)}=O\left(3^{9} \pi^{12 \nu}\right)$, and this yields $b=O\left(3^{-1.25} \pi^{v}\right)$ too.

Remark 4.10. In [BKSW20], the authors describe the arithmetic properties of models of Picard curves when $\operatorname{char}(K) \neq 3$.

\section{Characterization of Potentially Good hyperelliptic REDUCtion}

5.1. Characterization of toggle model in terms of invariants. Let $K$ be a discrete valuation field with valuation $v$, valuation $\operatorname{ring} \mathcal{O}$ and a uniformizer $\pi$. We now restrict to $k=\mathcal{O} /\langle\pi\rangle$ of characteristic $p=0$ or $p>7$. We start with the following observation.

Lemma 5.1. Let $Q \in \mathcal{O}\left[x_{1}, x_{2}, x_{3}\right]$ be a quadratic ternary form. Then the Dixmier-Ohno invariants of $Q^{2}$ are

$$
\begin{aligned}
\underline{\mathrm{DO}}\left(Q^{2}\right)=\left(I_{3}, \frac{1}{2^{2} \cdot 3^{2} \cdot 5} I_{3}^{2}, \frac{7^{2}}{2^{2} \cdot 3^{2}} I_{3}^{3}, \frac{7^{2}}{2^{2} \cdot 3 \cdot 5} I_{3}^{3}, \frac{7^{3}}{2^{2} \cdot 3^{4} \cdot 5} I_{3}^{4}, \frac{7^{2}}{2^{2} \cdot 3^{2}} I_{3}^{4}, \frac{5 \cdot 7^{3}}{2^{4} \cdot 3^{5}} I_{3}^{5},\right. \\
\left.\frac{7^{3}}{2^{4} \cdot 3^{2} \cdot 5^{2}} I_{3}^{5}, \frac{7^{4}}{2^{4} \cdot 3^{5}} I_{3}^{6}, \frac{7^{4}}{2^{4} \cdot 3^{3} \cdot 5^{2}} I_{3}^{6}, \frac{7^{4}}{2^{2} \cdot 3^{4} \cdot 5} I_{3}^{7}, \frac{7^{4}}{2^{4} \cdot 3^{2} \cdot 5} I_{3}^{7}, 0\right)
\end{aligned}
$$

where $I_{3}=5 /\left(2^{2} \cdot 3^{2}\right) \cdot D_{3}(Q)^{2}$ where $D_{3}$ is the discriminant of ternary quadratic forms.

In particular, if $Q$ is non-degenerate, we have

$$
\operatorname{DO}\left(Q^{2}\right)=\left(1: \frac{1}{180}: \frac{49}{36}: \frac{49}{60}: \frac{343}{1620}: \frac{49}{36}: \frac{1715}{3888}: \frac{343}{3600}: \frac{2401}{3888}: \frac{2401}{10800}: \frac{343}{1620}: \frac{2401}{720}: 0\right) .
$$

Remark 5.2. With Lemma 5.1, we are now in position to determine the reduction type over $K$ of a smooth plane quartic $F=0$ (at least for any DVR $\mathcal{O}$ for which one can solve polynomial equations over $k$ ). The extra ingredients needed come from the methods developed in [Kol97] and forthcoming [ES20]. Indeed, their algorithms compute the finite list $S$ of $\mathrm{PGL}_{3}(\mathcal{O})$-equivalent classes of forms over $\mathcal{O}$ which are $\mathrm{PGL}_{3}(K)$-equivalent to $F$ and with a discriminant of minimal valuation. Now, the discussion splits into two cases. Clearly, one has good quartic reduction if and only if there is a form in $S$ with a discriminant of valuation 0 (and in this case one has actually $\# S=1$ ).

Secondly, one has good hyperelliptic reduction if and only if at least one form $F_{0} \in S$ satisfies the conditions of Proposition 1.2. The reverse implication is straightforward. For the direct implication, we know that the curve admits a good toogle model $F_{0}=0$ over $K$ (Theorem 1.4). Its discriminant is not of 
valuation 0 but $I_{3}\left(F_{0}\right)$ is, as we have just seen ${ }^{3}$. This means that the valuation of the discriminant of $F_{0}$ is minimal and therefore $F_{0}$ belongs to the class of a form in $S$, which is also a good toogle model (the properties of being a good toogle model do not change by the action of $\mathrm{PGL}_{3}(\mathcal{O})$ ). Unlike the case of good quartic reduction, $S$ may contain several forms having either bad or good hyperelliptic reduction. For instance $F / \mathbb{Q}_{\pi}=0$ with $\pi \in \mathbb{Z}_{>5}$ where $F=\left(x_{2}^{2}-4 x_{1} x_{3}\right)^{2}+\pi^{4}\left(x_{1}^{4}+x_{3}^{4}\right)$ has good hyperelliptic reduction whereas $F\left(\pi^{2} x_{1}, \pi x_{2}, x_{3}\right) / \pi^{4}=0$ has bad reduction and both have a discriminant with minimal valuation.

Proposition 5.3. The curve $C$ potentially admits a toggle model if and only if any minimal representative of $\mathrm{DO}(F)$ reduces modulo $\pi$ to the invariants in equation (5.1).

Proof. The direct implication is clear. For the converse, using Proposition 3.13, and after a possible extension, we can assume that we start with a model $C: F=0$ with $F \in \mathcal{O}\left[x_{1}, x_{2}, x_{3}\right]$ and that $\bar{F}=0$ is a GIT-semi-stable quartic with minimal orbit. Since $\bar{F}=0$ has the same invariants as the square of a non-degenerate quadric, which has an infinite automorphism group, it is not GIT-stable. The quartic $\bar{F}=0$ is therefore in $X^{s s} \backslash X^{s}$. By [Mum77, p.49] and [Art09, Lem.1.4.iii], after possibly a finite extension of $K, \bar{F}$ is either equivalent to the square of the non-degenerate quadratic form $Q_{0}=\left(x_{2}^{2}-4 x_{1} x_{3}\right)$ or to the product of $Q_{0}$ with a quadratic form $Q$ such that $Q_{0}=0$ is tangent to $Q=0$ (the tangent line being, say, $x_{1}=0$ ). In the first case, this means exactly that $F$ is a toggle model. In the second case, we have

$$
\bar{F}=\left(x_{2}^{2}-4 x_{1} x_{3}\right)\left(a x_{1}^{2}+b x_{1} x_{2}+c x_{1} x_{3}+d x_{2}^{2}\right) \text { with }(a, b, c, d) \in k^{4} .
$$

Moreover, since $\mathrm{DO}(\bar{F})=\mathrm{DO}\left(Q_{0}^{2}\right)$, a computation shows that $c \neq 0$ and $c=-4 d$. Hence we can rewrite (up to a multiplicative constant) $\bar{F}=Q_{0}^{2}+Q_{0} x_{1}\left(a x_{1}+b x_{2}\right)$. We therefore see that

$$
F=Q_{0}^{2}+Q_{0} x_{1}\left(a x_{1}+b x_{2}\right)+\pi G
$$

with $G \in \mathcal{O}\left[x_{1}, x_{2}, x_{3}\right]$. Finally the change of variables $\left(x_{1}, x_{2}, x_{3}\right) \mapsto\left(\pi^{1 / 4} x_{1}, \pi^{1 / 8} x_{2}, x_{3}\right)$, transforms $F$ into

$$
\pi^{1 / 2} \cdot(Q_{0}^{2}+\pi^{1 / 8} \underbrace{\left(Q_{0} x_{1}\left(a \pi^{1 / 8} x_{1}+b x_{2}\right)+\pi^{3 / 8} G\left(\pi^{1 / 4} x_{1}, \pi^{1 / 8} x_{2}, x_{3}\right)\right)}_{G_{0}}) .
$$

Hence, over an extension of $K$, we find the required model $F_{0}=Q_{0}^{2}+\pi^{1 / 8} G_{0}$.

Since having potentially good hyperelliptic reduction implies the existence of a toggle model, Proposition 5.3 is often strong enough to rule out this scenario, as the following example shows.

Example 5.4. Let $K=\mathbb{Q}_{11}$ and consider the case $X_{12} / K: F=0$ from [KLL ${ }^{+} 18$, Sec.5]. The valuations of $\underline{\mathrm{DO}}(F)$ are $(0,0,0,1,0,0,2,0,1,0,0,0,9)$ and hence $\underline{\mathrm{DO}}(F)$ is a minimal representative. Since $v_{\mathrm{DO}}\left(J_{9}(F)\right)=v\left(J_{9}(F)\right)>0$, the list $\underline{\mathrm{DO}}(F)$ cannot reduce modulo $\pi$ to equation (5.1). Therefore $X_{12}$ does not have potentially good reduction.

For the excluded characteristics $p=5$ or 7 (and conjecturally 3 ), one could replace the invariants in DO by the HSOPs of Theorems 4.1 and 4.3 and still get a characterization of having potentially a toggle model. For $p=2$, we get a necessary condition for the curve to have potentially good hyperelliptic reduction. Let us illustrate this by resuming the case of $X_{1}$ from Example 4.5. We see for instance that the valuations at 5 of the HSOP for $\left(x_{2}^{2}-4 x_{1} x_{3}\right)^{2}$ are $(1,1,1,3,0,6, \infty)$ whereas the ones of $X_{1}$ are

\footnotetext{
${ }^{3}$ With the notation of Theorem 4.1, the same property holds replacing $I_{3}$ by $I_{24}^{(2)}$ (resp. $I_{3}^{(3)}$, resp. $J_{15}^{(5)}$ ) in characteristic 2 (resp. 3, resp. 5).
} 
$(1,1,0,2,1,2,12)$. We can conclude that $X_{1}$ has not potentially good reduction at 5 and also at 7 with the same arguments. However at 2 , the reduction of the HSOP of $X_{1}$ is the same as the ones of $\left(x_{2}^{2}-x_{1} x_{3}\right)^{2}$ and we cannot conclude on wether $X_{1}$ has potentially good hyperelliptic reduction at 2 or does not have potentially good reduction. Notice that we slightly change the conic here so it remains non-degenerate modulo 2 and apply Theorem 2.9. We shall see how to deal with the prime 2 in Example 5.14.

5.2. The equivariant map $b_{8}$ and proof of Theorem 1.10. The equivalence in Theorem 1.10 is more complicated to establish than the one in Theorem 1.9 as we must link the world of genus 3 nonhyperelliptic curves (as ternary quartic forms) and hyperelliptic curves (as binary octics). The starting point is a beautiful result from representation theory we will recall now.

Let $R$ be an integral domain and let $W$ (resp. $V$ ) be free $R$-modules of rank $n=2$ (resp. $n=3$ ). We identify binary (resp. ternary) forms with coefficients in $R$ of degree $d \geq 0$ with elements in $\operatorname{Sym}^{d}\left(W^{\vee}\right)$ (resp. $\operatorname{Sym}^{d}\left(V^{\vee}\right)$ ). The usual action of $\mathrm{GL}_{n}(R)$ on the left for $W$ (resp. $V$ ) induces a left action on $\operatorname{Sym}^{d}(W)$ (resp. $\operatorname{Sym}^{d}(V)$ ) and a right action on the forms, which we denote in both cases with a dot. An identification of $V$ with $\operatorname{Sym}^{2}(W)$ defines a morphism $h: \mathrm{SL}(W) \rightarrow \operatorname{SL}(V)$ and a linear morphism $b_{2 d}: \operatorname{Sym}^{d}\left(V^{\vee}\right) \rightarrow \operatorname{Sym}^{2 d}\left(W^{\vee}\right)$ which is equivariant for the previous action, i.e. $F \cdot h(T)=b_{2 d}(F) \cdot T$ for any $T \in \mathrm{SL}(W)$. The identification of $V$ with $\operatorname{Sym}^{2}(W)$ also induces an embedding of $\mathbb{P}^{1}$ into $\mathbb{P}^{2}$ whose image is given by a conic $Q_{0}=0$ with $Q_{0}$ a quadratic form with coefficients in $R$. The image of the morphism $h$ is contained in the subgroup $\mathrm{SO}\left(Q_{0}\right)$ of elements $T \in \mathrm{SL}_{3}(R)$ such that $Q_{0} \cdot T=Q_{0}$.

To make it concrete and keeping with the literature, when the characteristic of $R$ is not equal to 2 , we choose the following particular basis for $\operatorname{Sym}^{2}(W)$. Let $u, v$ (resp. $v_{1}, v_{2}, v_{3}$ ) be a basis of $W$ (resp. $V$ ) and $x, z$ the dual basis of $W^{\vee}$ (resp. $x_{1}, x_{2}, x_{3}$ the dual basis of $V^{\vee}$ ). We let $u^{2}, 2 u v, v^{2}$ be a basis for $\operatorname{Sym}^{2}(W)$. Then $b_{2 d}(F)=F\left(x^{2}, 2 x z, z^{2}\right), Q_{0}=x_{2}^{2}-4 x_{1} x_{3}$ and

$$
h\left(\begin{array}{ll}
a & b \\
c & d
\end{array}\right)=\left(\begin{array}{ccc}
a^{2} & 2 a b & b^{2} \\
a c & a d+b c & b d \\
c^{2} & 2 c d & d^{2}
\end{array}\right) .
$$

Particularizing to the case of a quartic form $F$, a simple computation shows that $b_{8}(F)$ contains valuable information on the reduction.

Lemma 5.5. Let $\mathcal{C} / \mathcal{O}: F=0$ with $F=Q_{0}^{2}+\pi^{2 s} G$ be a toggle model. The model $\mathcal{C}$ is a good toggle model if and only if $\overline{b_{8}(G)}=\bar{G}\left(x^{2}, 2 x z, z^{2}\right)$ has 8 distinct roots. Moreover an equation of $\overline{\mathcal{C}}$ is $y^{2}=\overline{b_{8}(G)}$.

Let $F=Q_{0}^{2}+\pi^{2 s} G$ be a toggle model for a smooth plane quartic $C$. The article [LRS18] gives relations between the Shioda invariants of $b_{8}(F)$ and the Dixmier-Ohno invariants of $F$. These expressions can be shorten considerably in our situation by looking at the first terms of their $\pi$-expansions. 
Proposition 5.6. Let $F=Q_{0}^{2}+\pi^{2 s} G$ be a toggle model. Let $f=b_{8}(G)=b_{8}(F) / \pi^{2 s}$. The Shioda invariants $j_{i}$ for $2 \leq i \leq 7$ satisfy the congruences $\pi^{2 s i} \cdot j_{i}(f)=\iota_{3 i}(F)+O\left(\pi^{2 s(i+1)}\right)$ where

$$
\begin{aligned}
\iota_{6}= & \frac{3^{2}}{2^{5} \cdot 5 \cdot 7}\left(I_{3}{ }^{2}-180 I_{6}\right), \\
\iota_{9}= & \frac{3^{5}}{2^{9} \cdot 5^{2} \cdot 7^{3}}\left(14 I_{3}^{3}-2520 I_{3} I_{6}-81 I_{9}+135 J_{9}\right), \\
\iota_{12}= & \frac{3^{3}}{2^{14} \cdot 5 \cdot 7^{3}} I_{3}\left(-32 I_{3}^{3}+14580 I_{3} I_{6}+261 I_{9}-495 J_{9}\right)+\frac{5^{2}}{2 \cdot 3 \cdot 7^{2}} \iota_{6}^{2}, \\
\iota_{15}= & \frac{3^{4}}{2^{16} \cdot 5^{2} \cdot 7^{5}} I_{3}\left(-592 I_{3}^{4}+30780 I_{3}{ }^{2} I_{6}+2601 I_{3} I_{9}-45 I_{3} J_{9}+7290000 I_{6}{ }^{2}-2430 J_{12}\right)+\frac{5^{2}}{3^{2} \cdot 7} \iota_{6} \iota_{9}, \\
\iota_{18}= & \frac{3^{8}}{2^{24} \cdot 5^{2} \cdot 7^{4}} I_{3}^{2}\left(-8 I_{3}{ }^{4}-14418 I_{3}{ }^{2} I_{6}-117 I_{3} I_{9}+423 I_{3} J_{9}+155520 I_{6}{ }^{2}-486 I_{12}\right) \\
& +\frac{17^{3}}{2^{6} \cdot 3^{2} \cdot 7^{3}} \iota_{6}{ }^{3}+\frac{3 \cdot 5}{2^{5}} \iota_{9}^{2}-\frac{17}{2^{3} \cdot 7} \iota_{6} \iota_{12}, \\
\iota_{21}= & \frac{3^{7}}{2^{44} \cdot 5^{7} \cdot 7^{5}} I_{3}^{2}\left(-128 I_{3}{ }^{5}+213912 I_{3}{ }^{3} I_{6}+2961 I_{3}{ }^{2} I_{9}-8541 I_{3}{ }^{2} J_{9}-18057600 I_{3} I_{6}{ }^{2}+12204 I_{3} I_{12}\right. \\
& \left.+810 I_{3} J_{12}-45360 I_{6} I_{9}+285120 I_{6} J_{9}-4860 I_{15}-540 J_{15}\right)+\frac{2 \cdot 5^{3}}{3^{3} \cdot 7^{2}} \iota_{6}^{2} \iota_{9}-\frac{13}{2 \cdot 3^{2}} \iota_{9} \iota_{12}-\frac{17}{2^{2} \cdot 3 \cdot 7} \iota_{6} \iota_{15} .
\end{aligned}
$$

Finally $\pi^{14 \cdot 2 s} D_{14}(f)=\iota_{42}(F)+O\left(\pi^{15 \cdot 2 s}\right)$ with

$$
\iota_{42}=\frac{3^{10}}{2^{18} \cdot 5^{5}} I_{3}^{5} I_{27}
$$

The previous proposition defines a list of 6 invariants for ternary quartics $\underline{\iota}=\left(\iota_{6}, \ldots, \iota_{21}\right)$ whose evaluation at a generic ternary quartic form $F$ induces a point in $\mathbb{P}^{(6,9,12,15,18,21)}$.

We can now prove the direct implication of Theorem 1.10.

Theorem 5.7 (see Theorem 1.10). Let $K$ be a discrete valuation field with valuation v, valuation ring $\mathcal{O}$ and a uniformizer $\pi$. Assume that its residue field $k$ has characteristic $p \neq 2,3,5,7$. Let $C / K$ be a smooth plane quartic defined by $F=0$. Then $C$ has potentially good hyperelliptic reduction if and only if

$$
v_{\mathrm{DO}}\left(I_{3}(F)\right)=0, \quad v_{\mathrm{DO}}\left(I_{27}(F)\right)>0 \text { and } v_{\iota}\left(I_{3}(F)^{5} I_{27}(F)\right)=0 .
$$

Under these conditions, one can also obtain an explicit equation for the special fiber.

Direct implication of Theorem 1.10. Let $F=Q_{0}^{2}+\pi^{2 s} G$ be a good toggle model corresponding to a stable model $\mathcal{C} / \mathcal{O}$ as in Proposition 1.2. One has that $v_{\mathrm{DO}}\left(I_{3}(F)\right)=v\left(I_{3}(F)\right)=0$ since $I_{3}(\bar{F})=320 / 9$. So $v_{\mathrm{DO}}\left(I_{27}(F)\right)=v\left(I_{27}(F)\right)>0$ since $\bar{F}=0$ is singular. Moreover, the reduction of $f=b_{8}(G)$ gives an equation for $\mathcal{C}_{k}: y^{2}=\bar{f}(x, z)$. This implies that $v\left(D_{14}(f)\right)=0$ and therefore we get the equality $v\left(\iota_{42}(F)\right)=14 \cdot 2 s+v\left(D_{14}(f)\right)=14 \cdot 2 s$. Since each of the $\iota_{3 i}(F)$ is divisible by $\pi^{2 s i}$, we get that $v_{\iota}\left(\iota_{42}(F)\right)=v_{\iota}\left(I_{3}(F)^{5} I_{27}(F)\right)=0$.

The proof of the converse implication will keep us busy till the end of this section. For a ternary quartic form $F \in \operatorname{Sym}^{4}\left(V^{\vee}\right)$, let us denote $\rho: \operatorname{Sym}^{4}\left(V^{\vee}\right) \rightarrow \operatorname{Sym}^{2}(V)$ the contravariant of degree 4 and order 2 defined in [Dix87]. If $T \in \mathrm{GL}_{3}(R)$, then $\rho$ satisfies the relation $\rho(F \cdot T)=\operatorname{det}(T)^{6} \cdot T^{-1} \cdot \rho(F)$. Let $\rho_{0}=\rho\left(Q_{0}^{2}\right)$. Then

$$
\rho_{0}=-2^{12} \cdot 3^{-2} \cdot 5 \cdot 7 \cdot\left(v_{2}^{2}-v_{1} v_{3}\right)
$$

Lemma 5.8. Suppose that $\mathcal{O}$ is complete. Let $\mathcal{W}$ be a smooth scheme over $\mathcal{O}$. Then for any $s \geq 1$, the canonical map

$$
\mathcal{W}(\mathcal{O}) \rightarrow \mathcal{W}\left(\mathcal{O} /\left(\pi^{s}\right)\right)
$$

is surjective. 
Proof. Indeed, for any $\sigma \in \mathcal{W}\left(\mathcal{O} /\left(\pi^{s}\right)\right)=\operatorname{Hom}_{\mathcal{O}}\left(\operatorname{Spec}\left(\mathcal{O} /\left(\pi^{s}\right)\right), \mathcal{W}\right)$ we can replace $\mathcal{W}$ by an affine open neighborhood of the image of $\sigma$ and it is enough to lift $\sigma$ there. So we can suppose $\mathcal{W}$ is affine. As $\mathcal{O}$ is complete, the canonical map

$$
\mathcal{W}(\mathcal{O}) \rightarrow \operatorname{proj} \lim _{n \geq s} \mathcal{W}\left(\mathcal{O} /\left(\pi^{n}\right)\right)
$$

is an isomorphism. By the smoothness of $\mathcal{W}$, for any pair of integers $n>r>0$, the canonical map $\mathcal{W}\left(\mathcal{O} /\left(\pi^{n}\right)\right) \rightarrow \mathcal{W}\left(\mathcal{O} /\left(\pi^{r}\right)\right)$ is surjective. So $\mathcal{W}(\mathcal{O}) \rightarrow \mathcal{W}\left(\mathcal{O} /\left(\pi^{s}\right)\right)$ is surjective.

Proposition 5.9. Suppose that $\mathcal{O}$ is complete. Let $\rho \in \mathcal{O}\left[v_{1}, v_{2}, v_{3}\right]$ be a quadratic (dual) form such that for some $s \geq 1$ we have $\rho \equiv \rho_{0} \bmod \pi^{s}$. Then there exists $T \in \mathrm{GL}_{3}(\mathcal{O})$ such that

$$
T \equiv \mathrm{Id}_{3} \quad \bmod \pi^{s}, \quad \text { T. } \rho=u \rho_{0} .
$$

with $u \in \mathcal{O}$ such that $u \equiv 1 \bmod \pi^{s}$.

Proof. Let $\mathcal{W}=\underline{\operatorname{Isom}}_{\mathcal{O}}\left(V_{+}(\rho), V_{+}\left(\rho_{0}\right)\right)$ be the scheme of linear isomorphisms. For any $\mathcal{O}$-algebra $A$, $\mathcal{W}(A)$ is the set of matrices $U=\left(x_{i j}\right)_{1 \leq i, j \leq 3}$ such that

$$
{ }^{t} U \rho U=y \rho_{0}, \quad y \in A^{*}
$$

(here we identify the quadratic forms with their matrices in the canonical basis). The scheme $\mathcal{W}$ is defined by 6 equations in the affine space of relative dimension 10 (9 for the entries of $U$ plus 1 for the ratio $y$ ). Its Jacobian matrix at the point $I: U=\operatorname{Id}_{3} \in M_{3 \times 3}(k), y=1$ has rank $4^{4}$. This implies that $\mathcal{W}$ is smooth at this point.

By hypothesis, there exists a $\sigma \in \mathcal{W}\left(\mathcal{O} /\left(\pi^{s}\right)\right)$ whose image in $\mathcal{W}(k)$ is equal to the point $I$ above. Applying the previous lemma to the smooth locus of $\mathcal{W}$ (in fact $\mathcal{W}$ is smooth everywhere), we find a lifting $T \in \mathcal{W}(\mathcal{O})$ of $\sigma$. Then $T \in \mathrm{GL}_{3}(\mathcal{O})$ satisfies

$$
T \equiv \operatorname{Id}_{3} \quad \bmod \pi^{s}, \quad T \cdot \rho=u \rho_{0}
$$

with $u \equiv 1\left(\bmod \pi^{s}\right)$. Thus $T$ satisfies the required properties.

Corollary 5.10. Suppose that $\mathcal{O}$ is complete and let $F=Q_{0}^{2}+\pi^{2 s} G$ be a toggle model. There exists a matrix $T_{1} \in \mathrm{GL}_{3}(\mathcal{O})$ with $T_{1} \equiv \mathrm{Id}_{3} \bmod \pi^{2 s}$ such that $F_{1}=F . T_{1}=Q_{0}^{2}+\pi^{2 s} G_{1}$ satisfies $\rho\left(F_{1}\right)=\rho_{0}$.

Proof. Let $T$ be the matrix of Proposition 5.9 with $\rho=\rho(F)$ and let $F^{\prime}=F \cdot T^{-1}$. Since one has $T^{-1} \equiv \operatorname{Id}_{3}\left(\bmod \pi^{2 s}\right)$, we see that $F^{\prime}=Q_{0}^{2}+\pi^{2 s} G^{\prime}$. Moreover $\rho\left(F^{\prime}\right)=\operatorname{det}(T)^{6} \cdot T \cdot \rho(F)=\operatorname{det}(T)^{6} \cdot u \cdot \rho_{0}$. Let now $\alpha=\left(\operatorname{det}(T)^{6} \cdot u\right)^{-1 / 4}$ with $\alpha \equiv 1\left(\bmod \pi^{2 s}\right)$. Then the form $F_{1}=\alpha F^{\prime}=F \cdot\left(\alpha T^{-1}\right)$ satisfies the hypotheses.

Proposition 5.11. Let $C / K: F=0$ with $F=Q_{0}^{2}+\pi^{2 s} G\left(x_{1}, x_{2}, x_{3}\right)$ be a toggle model of $C$ such that $\rho(F)=\rho_{0}$. If $b_{8}(G)$ is GIT-stable over $\bar{K}$, up to a possible extension of $K$, we can assume that $\overline{b_{8}(G)}$ is GIT-semi-stable.

Proof. Let $f=b_{8}(G)$. By Corollary 3.5, after a possible extension of $K$, there exists a matrix $T \in \mathrm{SL}_{2}(K)$ such that $f . T=\pi^{r} \cdot f_{1}$ with $r \in \mathbb{Z}$ and $f_{1} \in \mathcal{O}[x, z]$ such that $\bar{f}_{1}=0$ is GIT-semi-stable. Comparing the discriminants on both sides, we see that $r \geq 0$. Since the kernel of $b_{8}$ is $Q_{0} Q$ where $Q$ is any quadratic form, we can write $G . h(T)$ in a unique way as $Q_{0} Q_{1}+G_{1}$ with $Q_{1} \in K\left[x_{1}, x_{2}, x_{3}\right]$ a quadratic form and $G_{1}=\beta_{8} x_{1}^{4}+\beta_{7} x_{1}^{3} x_{2}+\beta_{6} x_{1}^{3} x_{3}+\beta_{5} x_{1} x_{2}^{3}+\beta_{4} x_{1} x_{2}^{2} x_{3}+\beta_{3} x_{2}^{3} x_{3}+\beta_{2} x_{2}^{2} x_{3}^{2}+\beta_{1} x_{2} x_{3}^{3}+\beta_{0} x_{3}^{4} \in K\left[x_{1}, x_{2}, x_{3}\right]$.

\footnotetext{
${ }^{4}$ For the explicit computations one can take for $\rho_{0}$ the sum of the squares $\sum_{i} v_{i}^{2}$ as the residue characteristic is different from 2 .
} 
Since

$$
\begin{aligned}
b_{8}\left(G_{1}\right) & =\beta_{8} x^{8}+2 \beta_{7} x^{7} z+\beta_{6} x^{6} z^{2}+8 \beta_{5} x^{5} z^{3}+4 \beta_{4} x^{4} z^{4}+8 \beta_{3} x^{3} z^{5}+4 \beta_{2} x^{2} z^{6}+2 \beta_{1} x z^{7}+\beta_{0} z^{8} \\
& =\pi^{r} f_{1},
\end{aligned}
$$

we get that $v\left(\beta_{i}\right) \geq r$ for all $i \in\{0, \ldots, 8\}$, in particular there exists $G_{2} \in \mathcal{O}\left[x_{1}, x_{2}, x_{3}\right]$ such that $G_{1}=\pi^{r} G_{2}$. Let us now write $Q_{1}=\pi^{t} Q_{2}$ with $t \in \mathbb{Z}$ and $Q_{2} \in \mathcal{O}\left[x_{1}, x_{2}, x_{3}\right]$ primitive. We have that

$$
F . h(T)=Q_{0}^{2}+\pi^{2 s} G . h(T)=Q_{0}^{2}+\pi^{2 s+t} Q_{0} Q_{2}+\pi^{2 s+r} G_{2} .
$$

Since $\rho(F . h(T))=\rho(F)=\rho_{0}$, Lemma 5.12 implies that $2 s+t \geq 2 s+r$ so $t \geq r$. Hence, we get that

$$
F . h(T)=Q_{0}^{2}+\pi^{2 s+r} \underbrace{\left(G_{2}+\pi^{t-r} Q_{0} Q_{2}\right)}_{G_{3}} .
$$

We have that $b_{8}\left(G_{3}\right)=b_{8}\left(G_{2}\right)=b_{8}\left(G_{1} / \pi^{r}\right)=f_{1}$. This last equality also implies that $G_{3}$ is primitive.

Lemma 5.12. Let $F=Q_{0}^{2}+\pi^{a} Q_{0} Q+\pi^{b} G \in K\left[x_{1}, x_{2}, x_{3}\right]$ be a ternary quartic form such that $a, b \in \mathbb{Z}$, $b>0, Q$ is an integral primitive quadric and $G$ is an integral quartic form. If $\rho(F) \equiv \rho_{0}\left(\bmod \pi^{a+1}\right)$, then $a \geq b$.

Proof. Assume $a<b$ and write $Q=a_{1} x_{1}^{2}+a_{2} x_{1} x_{2}+a_{3} x_{1} x_{3}+a_{4} x_{2}^{2}+a_{5} x_{2} x_{3}+a_{6} x_{3}^{2}$. We see that modulo $\pi^{a+1}$, the coefficients of $\rho(F)$ are equal to

$$
\begin{aligned}
& \left(\frac{17920}{9} \pi^{a} a_{6},-\frac{35840}{9} \pi^{a} a_{5},-\frac{89600}{9} \pi^{a} a_{4}+\frac{71680}{3} \pi^{a} a_{3}+\frac{143360}{9},\right. \\
& \left.\frac{35840}{3} \pi^{a} a_{4}-\frac{143360}{9} \pi^{a} a_{3}-\frac{143360}{9},-\frac{35840}{9} \pi^{a} a_{2}, \frac{17920}{9} \pi^{a} a_{1}\right) .
\end{aligned}
$$

The equality $\rho(F) \equiv \rho_{0} \quad\left(\bmod \pi^{a+1}\right)$ imposes that they should be congruent to

$$
\left(0,0, \frac{143360}{9},-\frac{143360}{9}, 0,0\right)
$$

modulo $\pi^{a+1}$, from where we easily check that $v\left(a_{i}\right) \geq 1$ for all $i \in\{1, \ldots, 6\}$ and we get a contradiction with $Q$ being primitive.

We can now finish the proof of Theorem 1.10.

Reverse implication of Theorem 1.10. Let us assume that

$$
v_{\mathrm{DO}}\left(I_{3}(F)\right)=0, v_{\mathrm{DO}}\left(I_{27}(F)\right)>0 \text { and } v_{\iota}\left(\iota_{42}(F)\right)=0 .
$$

By Proposition 3.13, after a possible extension of $K$, there exists a form $F_{0}$ that is $\mathrm{GL}_{3}(K)$-equivalent to $F$, integral and primitive, and such that $v\left(I_{3}\left(F_{0}\right)\right)=0$ and $v\left(I_{27}\left(F_{0}\right)\right)>0$. Since $v_{\iota}\left(\iota_{42}(F)\right)=$ $v_{\iota}\left(\iota_{42}\left(F_{0}\right)\right)=0$ and $v\left(\iota_{42}\left(F_{0}\right)\right)=5 v\left(I_{3}\left(F_{0}\right)\right)+v\left(I_{27}\left(F_{0}\right)\right)>0$, the condition $v_{\iota}\left(\iota_{42}(F)\right)=0$ imposes that $v\left(\iota_{3 i}\left(F_{0}\right)\right)>0$ for $i=2, \ldots, 7$. This yields these 6 equations that must be satisfied by $\bar{F}_{0}$ :

$$
I_{6}=\frac{1}{180} I_{3}^{2}, I_{9}=\frac{49}{36} I_{3}^{3}, J_{9}=\frac{49}{60} I_{3}^{3}, I_{12}=\frac{343}{1620} I_{3}^{4}, J_{12}=\frac{49}{36} I_{3}^{4}, J_{15}+9 I_{15}=\frac{2744}{675} I_{3}^{5} .
$$

Substituting these expressions in the 23 relations satisfied by the Dixmier-Ohno invariants [Ohn07], we obtain a polynomial system that decomposes into 5 distinct radical components. The three first ones are such that $I_{3}\left(\bar{F}_{0}\right)=0$, and the last one is such that $I_{27}\left(\bar{F}_{0}\right) \neq 0$, which in both cases contradicts our hypotheses. There thus only remains one case for which $\mathrm{DO}\left(\bar{F}_{0}\right)$ are equal to the ones in (5.1). We therefore get by Proposition 5.3 that $F$ admits a toggle model $Q_{0}^{2}+\pi^{2 r} G=0$.

Since the reduction type of the stable model does not change by completion [Liu02, Prop. 10.3.15] and neither do the assumptions, we can take $\mathcal{O}$ complete. Using Corollary 5.10, we can furthermore 
assume that $\rho(F)=\rho_{0}$. Moreover since $v_{\iota}\left(\iota_{42}(F)\right)=0$, equation (5.4) shows that $v\left(D_{14}\left(b_{8}(G)\right) \neq \infty\right.$, i.e. $D_{14}\left(b_{8}(G)\right) \neq 0$ and so $b_{8}(G)=0$ is GIT-stable over $\bar{K}$ (see [MF82, Prop.4.2]). We are therefore in the situation of Proposition 5.11. In particular, we know that there exists a toggle model $F_{1}=Q_{0}^{2}+\pi^{2 s} G_{1}$ such that $f_{1}=b_{8}\left(G_{1}\right)$ has GIT-semi-stable reduction. Hence, by Corollary 3.17, there exists $2 \leq i_{0} \leq 7$ such that $v\left(j_{i_{0}}\left(f_{1}\right)\right)=0$. From equation (5.3), we get that $v\left(\iota_{3 i_{0}}\left(F_{1}\right)\right)=\pi^{i_{0} \cdot 2 s}$. Hence

$$
\frac{\iota_{6}\left(F_{1}\right)}{\pi^{2 \cdot 2 s}}, \ldots, \frac{\iota_{21}\left(F_{1}\right)}{\pi^{7 \cdot 2 s}}, \frac{\iota_{42}\left(F_{1}\right)}{\pi^{14 \cdot 2 s}}
$$

is a minimal representative of the invariants $\iota$ of $F$. Since we have assume that $v_{\iota}\left(\iota_{42}(F)\right)=0$, this implies that $v\left(\iota_{42}\left(F_{1}\right)\right)=14 \cdot 2 s$, so $v\left(D_{14}\left(f_{1}\right)\right)=0$ and $F_{1}$ is a good toggle model, which concludes the proof.

Actually, one can get expressions as in Proposition 5.6 for all the Shioda invariants $j_{2}, \ldots, j_{10}$. In the case of potentially good hyperelliptic reduction, we can therefore reconstruct an equation of the special fiber over $\bar{k}$ using the results of [LR12].

Example 5.13. We resume with Example 1.1. Let $C / \mathbb{Q}: F=0$ where

$$
F=\left(x_{2}+x_{3}\right) x_{1}^{3}-\left(2 x_{2}^{2}+x_{3} x_{2}\right) x_{1}^{2}+\left(x_{2}^{3}-x_{3} x_{2}^{2}+2 x_{3}^{2} x_{2}-x_{3}^{3}\right) x_{1}-2 x_{3}^{2} x_{2}^{2}+3 x_{3}^{3} x_{2} .
$$

One can check that $D_{27}(F)=-13^{6}$ so the curve has good quartic reduction away from 13 . At 13 the valuations of Dixmier-Ohno invariants are all 0 except for the one of $D_{27}(F)$ which is 6 . Moreover using the formulas of Proposition 5.6, we get that the valuations at 13 of the invariants $\iota$ are $(1,2,2,3,3,3)$. Therefore

$v_{\mathrm{DO}}\left(I_{3}(F)\right)=0, v_{\mathrm{DO}}\left(D_{27}(F)\right)=\frac{6}{27}>0, v_{\iota}\left(I_{3}(F)^{5} D_{27}(F)\right)=\frac{6}{42}-\min \left\{\frac{1}{6}, \frac{2}{9}, \frac{2}{12}, \frac{3}{15}, \frac{3}{18}, \frac{3}{21}, \frac{6}{42}\right\}=0$.

The curve has hence potentially good hyperelliptic reduction at 13 and its special fiber is the hyperelliptic curve of affine equation $y^{2}=x^{7}-1$.

Example 5.14. We conclude with Example 4.5. Applying our criterion to the primes $p=37$ and $p=$ 15187, we find that $X_{1}$ has potentially good hyperelliptic reduction at these primes. Actually, at these primes it would be easy to write a good toggle model since $F_{1}$ is of the form $Q^{2}+p G$. In Example 5.4, we took care of the excluded primes 5 and 7 . For 2, one can apply [KLL ${ }^{+} 18$, Prop.4.1]: there are 2 primes over 2 in the maximal CM order of $X_{1}$, hence the Jacobian is absolutely simple and $X_{1}$ has potentially good hyperelliptic reduction at 2 .

Similar analyses can be made for all other $X_{i}$ from loc. cit.. We summarize in the Table 4 our best knowledge of the reduction type of each of them at the primes dividing their discriminant. When we had to use the CM order to reach a conclusion, we indicate it with a $\dagger$.

Remark 5.15. The assumption $p>7$ was used in various occasions. In Lemma 5.1 or in Proposition 5.6, a better choice of invariants or normalization of the expressions may overcome this restriction, but the appearance of these primes in equation (5.5) is more symptomatic. Let us for instance consider $p=7$. It is known that the Klein quartic $C / \mathbb{Q}: F=0$ where $F=x_{1}^{3} x_{2}+x_{2}^{3} x_{3}+x_{3}^{3} x_{1}$ has potentially good hyperelliptic reduction modulo 7. However, since $\operatorname{Aut}_{\overline{\mathbb{Q}}}(C) \simeq \mathrm{PSL}_{2}\left(\mathbb{F}_{7}\right)$ and the latter is not a subgroup of $\mathrm{SO}_{3}(\overline{\mathbb{Q}}) \simeq \mathrm{PSL}_{2}(\overline{\mathbb{Q}})$, it cannot be the automorphism group of any non-degenerate conic. Hence, $\rho_{0}(F)=0$ and this is the case for any other possible covariant or contravariant of order 2. Therefore, we cannot go further with our current strategy in this case. However, in this particular case it is easy to find a toggle model, see [Elk99, p.56 and p.82]. 


\begin{tabular}{|c|c|c|c|c|}
\hline Curve & Pot. non-hyp. & Pot. hyp. & No pot. good & Unknown \\
\hline$\overline{X_{1}}$ & 13 & $2^{\dagger}, 37,15187$ & 5,7 & \\
\hline$X_{2}$ & & $2^{\dagger}, 701$ & 3,7 & \\
\hline$X_{3}$ & 31 & $2^{\dagger}, 233,356399$ & 3,5 & 7 \\
\hline$X_{5}$ & 13 & $2^{\dagger}, 37,127$ & 3 & 7 \\
\hline$X_{6}$ & 19 & $2^{\dagger}, 127,211,20707$ & 3,17 & 7 \\
\hline$X_{7}$ & 73 & $2^{\dagger}, 71,17665559$ & 3,83 & 7 \\
\hline$X_{8}$ & 19 & $2^{\dagger}, 499$ & 7 & \\
\hline$X_{9}$ & 13 & $79,233,857$ & 5 & 2,7 \\
\hline$X_{10}$ & & 41,71 & & 2,7 \\
\hline$X_{11}$ & 31 & $23,47,27527$ & & 2,7 \\
\hline$X_{12}$ & & 5711,73064203493 & 11 & 7 \\
\hline$X_{13}$ & 43 & $547,11827,189169$ & 2,11 & \\
\hline$X_{14}$ & 19 & $101,107,8378707$ & 11 & \\
\hline$X_{15}$ & & 19 & & \\
\hline$X_{16}$ & & $19,37,79,13373064392147$ & 3 & \\
\hline$X_{17}$ & & $19,1229,3913841117$ & 2 & 3 \\
\hline$X_{18}$ & 13 & $19,101,251,7468843725186901$ & 2 & \\
\hline$X_{19}$ & & 11,43 & 2 & \\
\hline$X_{20}$ & & $67,1439,2739021126001$ & & \\
\hline
\end{tabular}

TABLE 4. CM-curves from $\left[\mathrm{KLL}^{+} 18\right]$ with the reduction type for the primes dividing the discriminant

\section{REFERENCES}

[ACGH85] E. Arbarello, M. Cornalba, P. Griffiths, and J. Harris. Geometry of algebraic curves, Vol. I, volume 267. Grundlehren der Mathematischen Wissenschaften, Springer-Verlag, New-York, 1985.

[Art09] M. Artebani. A compactification of $M_{3}$ via K3 surfaces. Nagoya Math. J., 196:1-26, 2009.

[Bas15] R. Basson. Arithmétique des espaces de modules des courbes hyperelliptiques de genre 3 en caractéristique positive. PhD thesis, Université de Rennes 1, Rennes, 2015.

[BBW17] M. Börner, I. I. Bouw, and S. Wewers. Picard curves with small conductor. In Algorithmic and experimental methods in algebra, geometry, and number theory, pages 97-122. Springer, Cham, 2017.

$\left[\mathrm{BCL}^{+} 15\right]$ I. Bouw, J. Cooley, K. Lauter, E. Lorenzo García, M. Manes, R. Newton, and E. Ozman. Bad reduction of genus three curves with complex multiplication. In Women in numbers Europe, volume 2 of Assoc. Women Math. Ser., pages 109-151. Springer, Cham, 2015.

$\left[\mathrm{BDM}^{+} 19\right]$ J. Balakrishnan, N. Dogra, J. S. Müller, J. Tuitman, and J. Vonk. Explicit Chabauty-Kim for the split Cartan modular curve of level 13. Ann. of Math. (2), 189(3):885-944, 2019.

[BKSW20] I. I. Bouw, A. Koutsianas, J. Sijsling, and S. Wewers. Conductor and discriminant of picard curves, 2020. To appear in J. of the London Math. Soc.

[Bur92] J.-F. Burnol. Remarques sur la stabilité en arithmétique. Internat. Math. Res. Notices, (6):117-127, 1992.

[BW17] I. I. Bouw and S. Wewers. Computing L-functions and semistable reduction of superelliptic curves. Glasg. Math. J., 59(1):77-108, 2017.

[Cle80] C. H. Clemens. A scrapbook of complex curve theory. Plenum Press, New York-London, 1980. The University Series in Mathematics.

[DDMM19] T. Dokchitser, V. Dokchitser, C. Maistret, and A. Morgan. Semistable types of hyperelliptic curves. In Algebraic curves and their applications, volume 724 of Contemp. Math., pages 73-135. Amer. Math. Soc., Providence, RI, 2019.

[Dem12] M. Demazure. Résultant, discriminant. Enseign. Math. (2), 58(3-4):333-373, 2012.

[Dix87] J. Dixmier. On the projective invariants of quartic plane curves. Adv. in Math., 64:279-304, 1987.

[DK02] H. Derksen and G. Kemper. Computational invariant theory. Invariant Theory and Algebraic Transformation Groups, I. Springer-Verlag, Berlin, 2002. Encyclopaedia of Mathematical Sciences, 130.

[Edi90] B. Edixhoven. Minimal resolution and stable reduction of $X_{0}(N)$. Ann. Inst. Fourier, 40(1):31-67, 1990.

[Elk99] N. D. Elkies. The Klein quartic in number theory. Math. Sci. Res. Inst. Publ., 35:51-101, 1999. 
[Els15] A.-S. Elsenhans. Explicit computations of invariants of plane quartic curves. J. Symbolic Comput., 68(part 2):109-115, 2015.

[ES20] A.-S. Elsenhans and M. Stoll. Good models for cubic surfaces. In progress, 2020.

[GKZ94] I. M. Gelfand, M. M. Kapranov, and A. V. Zelevinsky. Discriminants, resultants, and multidimensional determinants. Mathematics: Theory \& Applications. Birkhäuser Boston Inc., Boston, MA, 1994.

[Gla79] J. P. Glass. Bitangents of plane quartics. Bull. Austral. Math. Soc., 20(2):207-210, 1979.

[GLL15] O. Gabber, Q. Liu, and D. Lorenzini. Hypersurfaces in projective schemes and a moving lemma. Duke Math. J., 164(7):1187-1270, 2015.

[Har87] J. Harris. Curves and their moduli. In Algebraic geometry, Bowdoin, 1985 (Brunswick, Maine, 1985), volume 46 of Proc. Sympos. Pure Math., pages 99-143. Amer. Math. Soc., Providence, RI, 1987.

[Has99] B. Hassett. Stable log surfaces and limits of quartic plane curves. Manuscripta Math., 100(4):469-487, 1999.

[HL10] D. Hyeon and Y. Lee. Log minimal model program for the moduli space of stable curves of genus three. Math. Res. Lett., 17(4):625-636, 2010.

[HLP00] E. W. Howe, F. Leprévost, and B. Poonen. Large torsion subgroups of split Jacobians of curves of genus two or three. Forum Math., 12(3):315-364, 2000.

[HM98] J. Harris and I. Morrison. Moduli of curves, volume 187 of Graduate Texts in Mathematics. Springer-Verlag, New York, 1998.

[Hol95] R.-P. Holzapfel. The ball and some Hilbert problems. Lectures in Mathematics ETH Zürich. Birkhäuser Verlag, Basel, 1995. Appendix I by J. Estrada Sarlabous.

[Kan00] P.-L. Kang. On singular plane quartics as limits of smooth curves of genus three. J. Korean Math. Soc., 37(3):411-436, 2000.

[KlcLGS20] P. n. Kı lıçer, E. Lorenzo García, and M. Streng. Primes dividing invariants of CM Picard curves. Canad. J. Math., 72(2):480-504, 2020.

$\left[\mathrm{KLL}^{+}{ }^{16}\right]$ P. Kılıçer, K. Lauter, E. Lorenzo García, R. Newton, E. Ozman, and M. Streng. A bound on the primes of bad reduction of CM curves of genus 3. 2016.

$\left[\mathrm{KLL}^{+}{ }^{18}\right]$ P. Kılı̧̧er, H. Labrande, R. Lercier, C. Ritzenthaler, J. Sijsling, and M. Streng. Plane quartics over $\mathbb{Q}$ with complex multiplication. Acta Arith., 185(2):127-156, 2018.

[Kol97] J. Kollár. Polynomials with integral coefficients, equivalent to a given polynomial. Electron. Res. Announc. Amer. Math. Soc., 3:17-27, 1997.

[Kon00] S. Kondō. A complex hyperbolic structure for the moduli space of curves of genus three. J. Reine Angew. Math., 525:219-232, 2000.

[KW05] K. Koike and A. Weng. Construction of CM Picard curves. Math. Comp., 74(249):499-518 (electronic), 2005.

[Liu93] Q. Liu. Courbes stables de genre 2 et leur schéma de modules. Math. Ann., 295(2):201-222, 1993.

[Liu02] Q. Liu. Algebraic geometry and arithmetic curves, volume 6 of Oxford Graduate Texts in Mathematics. Oxford University Press, Oxford, 2002. Translated from the French by Reinie Erné, Oxford Science Publications.

[LL99] Q. Liu and D. Lorenzini. Models of curves and finite covers. Compositio Math., 118:61-102, 1999.

[LLLR19] R. Lercier, Q. Liu, E. Lorenzo García, and C. Ritzenthaler. Reduction type of smooth quartics, ancillary files. Available at https://arxiv.org/src/1803.05816/anc/, 2019.

[LR12] R. Lercier and C. Ritzenthaler. Hyperelliptic curves and their invariants: geometric, arithmetic and algorithmic aspects. J. Algebra, 372:595-636, 2012.

[LR19] R. Lercier and C. Ritzenthaler. Siegel modular forms of degree three and invariants of ternary quartics. Proc. Amer. Math. Soc., 2019. To appear.

[LRS18] R. Lercier, C. Ritzenthaler, and J. Sijsling. Reconstructing plane quartics from their invariants. Discrete 86 Computational Geometry, pages 1-41, 2018.

[LS16] J.-C. Lario and A. Somoza. A note on Picard curves of CM-type. Preprint, 2016.

[MF82] D. Mumford and J. Fogarty. Geometric invariant theory, volume 34 of Ergebnisse der Mathematik und ihrer Grenzgebiete [Results in Mathematics and Related Areas]. Springer-Verlag, Berlin, second edition, 1982.

[Mum77] D. Mumford. Stability of projective varieties. Enseignement Math. (2), 23(1-2):39-110, 1977.

[Ohn07] T. Ohno. The graded ring of invariants of ternary quartics I, 2007. unpublished.

[Ses77] C. S. Seshadri. Geometric reductivity over arbitrary base. Advances in Math., 26(3):225-274, 1977.

[Sha80] J. Shah. A complete moduli space for K3 surfaces of degree 2. Ann. of Math. (2), 112(3):485-510, 1980.

[Shi67] T. Shioda. On the graded ring of invariants of binary octavics. American J. of Math., 89(4):1022-1046, 1967. 


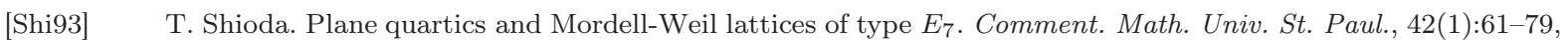
1993.

[Sil98] J. H. Silverman. The space of rational maps on $\mathbf{P}^{1}$. Duke Math. J., 94(1):41-77, 1998.

[STW14] L. Szpiro, M. Tepper, and P. Williams. Semi-stable reduction implies minimality of the resultant. J. Algebra, 397:489-498, 2014.

[Tsu86] S. Tsuyumine. On Siegel modular forms of degree three. Amer. J. Math., 108(4):755-862, 1986.

[Yuk86] A. Yukie. Applications of Equivariant Morse Stratifications (Geometric Invariant Theory, Eisenstein Series). ProQuest LLC, Ann Arbor, MI, 1986. Thesis (Ph.D.)-Harvard University.

Reynald Lercier, DGA \& Univ Rennes, CNRS, iRmaR - UMr 6625, F-35000 Rennes, France.

E-mail address: reynald.lercier@m4x.org

Qing Liu, Institut de mathématiques de Bordeaux, CNRS - UMR 5251, Université de Bordeaux, F-33405 Talence, France.

E-mail address: qing.liu@u-bordeaux.fr

Elisa Lorenzo García, Univ Rennes, CNRS, irmar - UMR 6625, F-35000 Rennes, France.

E-mail address: elisa.lorenzogarcia@univ-rennes1.fr

Christophe Ritzenthaler, Univ Rennes, CNRS, iRmaR - UMR 6625, F-35000 Rennes, France.

E-mail address: christophe.ritzenthaler@univ-rennes1.fr 